\title{
Quantum Speedup for Aeroscience and Engineering
}

\author{
Peyman Givi, ${ }^{*}$ Andrew J. Daley, ${ }^{\dagger}$ Dimitri Mavriplis, ${ }^{\dagger}$ and Mujeeb Malik ${ }^{\S}$
}

\begin{abstract}
Algorithms and hardware for quantum computing (QC) are reaching a critical stage in their development and have the potential to generate a paradigm shift in computing capability across a range of fields. Opportunities are growing for genuine impact of these systems over a timescale of 10-15 years, and there has been significant investment both from government agencies and private industry in the development of quantum computing. However, utilization of quantum phenomena is extraordinarily challenging due to its delicate nature and difficulties in measurement and control. A clear path exists towards demonstrating the advantages of QC over existing high-performance computing for some physics and materials science problems, but addressing practical computational challenges in other fields - though promising - is at an early stage of development. Reaching the next level of development will require strategic coordination between physicists, computer \& information scientists, mathematicians, and engineers, in order to transition this technology from the laboratory to robust and scalable computations for practical problems, especially those of interest to the aeroscience and engineering community. This community has been relying on high performance computing heavily, and will surely want to be informed of the developments in QC. This survey introduces the background and current state-of-the-art in QC, as well as its perceived opportunities and challenges.
\end{abstract}

\footnotetext{
*Professor, Mechanical Engineering and Petroleum Engineering, University of Pittsburgh, Fellow, AIAA.

$\dagger$ Professor, Physics and SUPA, University of Strathclyde.

†rofessor, Mechanical Engineering, University of Wyoming, Associate Fellow, AIAA.

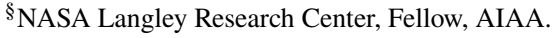




\section{Authors}

Peyman Givi is Distinguished Professor and James T. MacLeod Professor of Mechanical Engineering and Petroleum Engineering at the University of Pittsburgh. Previously he held the position of University at Buffalo Distinguished Professor of Aerospace Engineering. He has had frequent visiting appointments at the NASA Langley \& Glenn centers, and also worked as a Research Scientist at Flow Research Company. He is currently the Deputy Editor of AIAA Journal, and also serves on the editorial boards of Combustion Theory and Modelling, Computers \& Fluids, and Journal of Applied Fluid Mechanics. He is a recipient of the NASA Public Service Medal (2005), and is a Fellow of the AIAA.

Andrew Daley is Professor of Theoretical Quantum Optics at the University of Strathclyde, in Glasgow, UK. Prior to taking up this Chair, he held a faculty position at the University of Pittsburgh, after being a senior researcher at the Institute for Quantum Optics and Quantum Information of the Austrian Academy of Sciences in Innsbruck, Austria. He is currently the Principal Investigator of a UK Engineering and Physical Sciences Programme Grant in quantum simulation, and in the leadership team of the EU Quantum Technologies Flagship project on Programmable Atomic Large-Scale Quantum Simulation.

Dimitri Mavriplis is the Max Castagne Professor of Mechanical Engineering at the University of Wyoming. Prior to his position in academia, he spent 16 years at ICASE, co-located at the NASA Langley Research Center, where he worked on the development of unstructured mesh discretizations and solvers for computational aerodynamics. His current research group focuses on multidisciplinary analysis and optimization techniques as well as large scale aerodynamic simulations on emerging high-performance computing hardware. He is a co-author of the NASA sponsored CFD Vision 2030 report, serves as vice-chair of the AIAA CFD Vision 2030 Integration Committee, and is an Associate Fellow of the AIAA.

Mujeeb Malik is Senior Aerodynamicist at NASA's Langley Research Center, where he leads computational and experimental research for solving aeroscience challenge problems. He also sponsored NASA's CFD Vision 2030 Study and is the Founding Chair of AIAA CFD Vision 2030 Integration Committee. Previously, he served as Head of

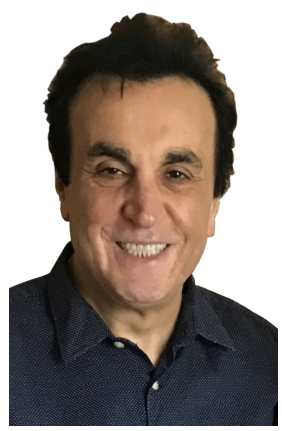

(a)

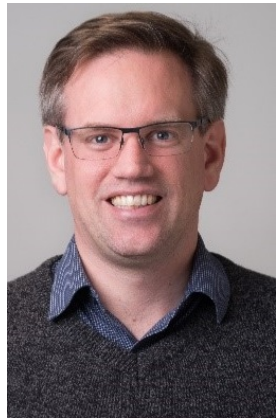

(b)

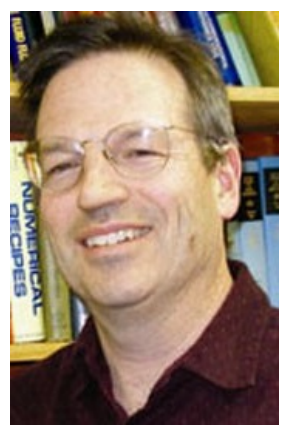

(c)

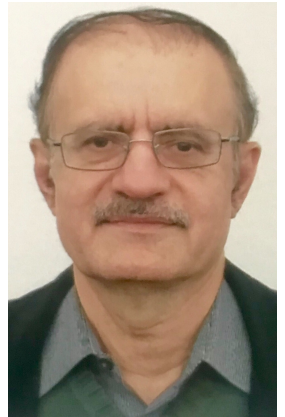

(d)

From Left: Peyman Givi, Andrew J. Daley, Dimitri Mavriplis, and Mujeeb Malik. 
Computational AeroSciences Branch at NASA LaRC. He is a recipient of the NASA Exceptional Service Medal (2012), and the NASA Silver Achievement Medal (2018). He is a Fellow of the AIAA. 


\section{Introduction}

OORE's law [1] has largely held true for over five decades. However, over the last few years, there has been
increasing discussion about its continued longevity as illustrated in Fig. [1] [2]. As silicon processes shrink to smaller and smaller sizes, physical limitations start to play an important role. As a result, the expense and effort required to continue the increase in performance are now much greater than ever before. In order to provide new disruptive means to perform computations with increased complexity, high performance computing will need a radical departure from the conventional classical computing platform. Quantum computing (QC) - which makes use of the unusual physical properties of microscopic objects to process information - is a particularly promising candidate to be this disruptive technology for a range of computational problems [3] 12$]$.

\section{A. Background}

Quantum Computing is one branch of the more general fields of Quantum Information Science (QIS) and Quantum Technology. QIS represents the merger of the two most significant scientific and technological revolutions of the 20th century, notably quantum physics and information technology. The resulting devices have the potential to revolutionize applications in sensing and metrology, communication, simulation and computing. Realization of the enormous scientific, economic and security implications of quantum technologies has led to rapidly growing investment worldwide, both from national governments in the US and abroad, and from private enterprises, as we detail below.

Quantum computing as an area has undergone rapid development, both in hardware and software, over the last decade. Used in appropriate ways, quantum mechanics can provide powerful resources for solving certain classes of problems, achieving cost scalings with the size of the problem that are not available to existing "classical" computers [10, 13]. This is known as"quantum speedup." Amongst the oldest and best known examples of quantum algorithms are Shor's algorithm for factorization of integers [14, 15], and Grover's algorithm for unstructured search problems [16]. The gain in efficiency of the scaling of these algorithms can either be exponential (i.e., a problem where the solution time on a classical computer scales exponentially in the size of the problem $N$ can have a solution time that scales polynomially in that size on a quantum computer); or polynomial (i.e., the problem scales polynomially with $N$ on a classical computer, and with a smaller power of $N$ on a quantum computer) [17, 18]. In either case, for the solution of large-scale problems for which quantum algorithms have been developed, quantum computers represent a potentially transformative new paradigm in computing.

Within the last few years, much progress has been made in experimental realizations of quantum computing hardware. Several architectures have been proposed based on a variety of physical hardware. On a small scale, quantum information 
has been stored and manipulated in a range of devices, including superconducting quantum bits $\left.\left.\right|^{2}\right]^{3}[19-\sqrt{27}$, trapped atomic ions [24, 28-34], neutral atoms [35,-39], silicon quantum electronics [40], electron spins [41-44], nuclear spins in the liquid or solid state [32, 45], and photons [46]. On the theoretical side, new quantum algorithms have recently been found, exhibiting significant polynomial speedups on quantum computers for solutions of a wide variety of problems. Examples include solution of linear equations [47-50], differential equations [48, 51, 51-57], quantum Monte Carlo simulation [58], and annealing problems [56], amongst a wide range of other possibilities [59].

The progress is very substantial across each of these hardware platforms, as we will review below, with challenges being systematically addressed on a path towards universal quantum computation and demonstration of quantum speedup. However, the developments in the last few years have also gone well beyond just progress towards universal quantum computing. There is a growing realization that the devices being developed may have short to medium term applications, especially in situations where the hardware can be tailored towards specific problems. This includes a range of approaches involving hybrid classical-quantum algorithms [60-62], and analogue devices such as quantum simulators [63, 64], and quantum annealers [65], where it might be possible to demonstrate quantum speedup [66]. Some of these devices already make it possible to study problems from quantum physics at a scale that would be intractable to classical high-performance computing [63]. The challenge is now to adapt the control over these devices, and improve certification techniques, in order to tailor them for solution of problems that are of interest beyond basic science in physics. Because the use of the hardware is specific to the problem being studied, this naturally must involve interdisciplinary collaborations with the end users of the hardware, with clear ties into mathematics, computer science, and engineering. This provides potential opportunities for the aerospace community and members of a broad range of other fields to contribute directly to the development of quantum computing for computational problems of interest in their own field.

\section{B. Investment in QC}

Internationally, there is very strong investment in QC both from governments and from private enterprise. This is strongly represented in the European Quantum Technologies Flagship, where projects are funded in Quantum Computing and Quantum Simulation pillars, in one of the United Kingdom's National Quantum Technologies hubs, as well as similarly sized investments through programs in China, Australia, Germany and the European Union [11].

In the US, various government agencies have initiated significant investments in QIS and an Interagency Working group on Quantum Information Science has been formed to coordinate research and investments across the federal government [67]. The Department of Energy (DOE), the Department of Defense and the National Science Foundation

\footnotetext{
${ }^{1}$ International Business Machines Corporation, IBM Builds its Most Powerful Universal Quantum Computing Processors, 2017, https://www03.ibm.com/press/us/en/pressrelease/52403.wss

${ }^{2}$ International Business Machines Corporation, IBM Building First Universal Quantum Computers for Business and Science, 2017, https://www03.ibm.com/press/us/en/pressrelease/51740.wss

${ }^{3}$ Rigetti Corporation, We are Close to a Universal Quantum Computer, Here is Where We are At, 2018, https://www.rigetti.com/news, https://youtu.be/6yaY4Fw-ovM
} 
have allocated significant resources to develop the hardware platforms and to assess the potential capabilities of quantum computing. In 2015, DOE sponsored a workshop to assess the extent of QC's capabilities to meet its computational requirements [68]. The promising outlook encouraged another workshop in 2017 focused on testbeds and the technologies needed to advance QC for actual applications within the next 5 years [13, 69]. A review of these programs suggests an exciting future in QC applications in a variety of problems. Within NASA, the Quantum Artificial Intelligence Laboratory (QUAIL) serves as a focal point for assessing the potential of quantum computers to impact computational challenges faced by the agency in the coming years [70]. There is also significant recent interest in national laboratories, publicly-traded companies, private industry and several recent start-up companies ${ }^{4}$

\section{Opportunities for Aeroscience Applications}

There is growing evidence that QIS in general, and QC in particular are approaching an inflection point, with significant opportunities and challenges for various scientific and engineering fields. In the fields of aeroscience and engineering, there are important implications for various national missions and responsibilities, including mission planning, autonomy, air space management, and material design - and advancing current high performance computing (HPC) applications such as computational materials research, computational fluid dynamics (CFD), combustion, aerothermodynamics and multidisciplinary design and optimization (MDAO). The CFD Vision 2030 report commissioned by NASA [71] advocates the need for continuous advances in HPC in the context of CFD and design optimization, notes the revolutionary impact that advances in quantum computing may have in these areas, and emphasizes the need to carefully monitor advances in this field as they develop. Given the potential of these new and emerging technologies, the aerospace community has a need to stay at the forefront of developments in quantum information science and quantum computing in order to know how and when to make strategic investments in these technologies and to accelerate their deployment into the most promising aerospace application areas.

\section{Purpose and Structure of This Survey}

Within the past 30 years, there has been impressive progress in developments of quantum computers and algorithms suitable for such computing. However, many open questions remain in regards to the future utilization of QC in relation to classical computing for engineering simulations. The objective of this survey is to give an overview of the state-of-the art in QC, tailored towards interests in the aerospace community. We begin with an introduction to the principles of how quantum computing works (\$[1], followed by a summary of the challenges in implementing universal quantum computing and an assessment of the state of the field in terms of hardware and software development (\$III). We describe both the progress in universal quantum computing as well as the potential for practical nearer-term applications via advances in analogue and special-purpose quantum simulators $(\$ \mathrm{IV})$. We then turn our focus to the potential applications

\footnotetext{
${ }^{4}$ Quantum Computing Report, https://quantumcomputingreport.com/players/
} 
of specific interest to aeroscience and engineering $(\$ \sqrt{\mathrm{V}})$, and future prospects $(\$ \sqrt{\mathrm{VI}})$.

\section{Universal Quantum Computing and Quantum Speedup}

In the late 1970s-1980s, quantum computing was discussed in different forms by a number of authors, inspired by the control that was gradually being developed over quantum systems. It was famously conceptualized by Feynman in a presentation in 1981 [72] as a potential means to simulate quantum mechanical systems, overcoming the exponential complexity for such simulations on a classical computer. The idea was formalized by Deutsch [73], and Bernstein and Vazirani [74] who further developed the modern concept of quantum computers, which are capable of solving problems that do not have efficient solutions on classical Church-Turing [75] machines.

The potential advantages arise from the quantum nature of QC processors, which can exist in many classically possible states simultaneously. This is similar to the behavior of matter on a microscopic or nanoscale level. Quantum computers take advantage of this so-called superposition of different states to gain a speedup; not just for simulating microscopic systems, but also for solving broad classes of classical computational problems. Initially this seems to be an overwhelming advantage, as quantum computers could simultaneously encode every possible piece of information that could be represented on it or a similarly-sized classical computer - an amount of information that grows exponentially with the size of the computer. However, there is a crucial difficulty in programming a quantum computer to take advantage of this opportunity. For example, while quantum computers can supposedly operate with superpositions of many states, at the end of the computation, when the final state is measured or output, the result collapses to a single classical state with a defined probability. That is, while QC makes use of exponentially large amounts of information, the inputs and outputs involve a reduction to the same amount of information that would be available on a classical computer of the same size. Additionally, exponentially many operations cannot be performed during the calculation. It is then a non-trivial task to design computational algorithms that will make use of the superposition of exponentially large amounts of information during a calculation, but manipulate it with few operations and reduce the output to a single useful answer or state.

To elaborate on this superposition of information, we describe in more detail how information is represented in a quantum computer. A qubit (sometimes written as qbit), or quantum bit, is the unit of quantum information [76]. It is the quantum analogue of the classical binary bit, but behaves markedly differently. In classical computing, the basic unit is a bit, which takes on the discrete values 0 or 1 . Calculations are done via logic gates using Boolean algebra. In quantum computing the base units are qubits, which have the quantum mechanical property of being simultaneously in both states 0 and 1 i.e., a superposition (Fig. 22). As noted above, when we measure or read out the state of a qubit, it collapses to the classical values of 0 or 1 with a prescribed probability. As is the standard in quantum physics, we use so-called Dirac notation in which a state $|\phi\rangle$ can be associated with a column vector $\phi$ of complex numbers. A qubit's state can be in any linear superposition of $|0\rangle$ and $|1\rangle$, i.e. $|\Psi\rangle=a_{0}|0\rangle+a_{1}|1\rangle$, where the complex numbers $a_{0}$ and $a_{1}$ 
are normalized to unity: $\left|a_{0}\right|^{2}+\left|a_{1}\right|^{2}=1$. This implies that the sum of the probabilities of the two states must be $100 \%$. The difference between the two means of computing is recognized by noting that a classical bit is specified either as a 0 or 1 , whereas a quantum bit (qubit) is specified by a "continuum" of the values of $a_{0}$ and $a_{1}$, with the requirement that the total probability of being in the two states is 1 .

As the number of qubits in a machine is increased, each of them can be entangled with some or all of the others. This implies that the state of any of the qubits can be correlated with the states of other qubits within the system in ways that would not be possible in classical physics. This "entanglement," makes it possible to have an arbitrary superposition involving any combination of the exponentially large number of classical configurations of all of the qubits. The corresponding exponentially large space is the basis of the potential advantages provided by a quantum computer - with the right algorithm for the right application, to double the computational power we only need to add a single qubit to the machine. The state of $N$ qubits is represented in Dirac notation [77] as:

$$
\begin{gathered}
|\Psi\rangle=\sum_{\ell=0}^{N-1} a_{\ell}|\ell\rangle=a_{0}|0 \ldots 00\rangle+a_{1}|0 \ldots 01\rangle+\ldots+a_{N-1}|1 \ldots 11\rangle, \\
\\
\left|a_{0}\right|^{2}+\left|a_{1}\right|^{2}+\ldots\left|a_{N-1}\right|^{2}=1,
\end{gathered}
$$

where the $a_{n}$ 's are complex numbers.

The most desired way of conducting quantum computation is via a gate-based digital computer, similar to that in classical computing. A quantum computer is composed of an ensemble of coupled qubits that are manipulated to implement a quantum algorithm in such a way that the final measurement provides that solution in the form of a probabilistic answer with a high degree of fidelity. The implementation of a quantum algorithm involves three basic steps, as depicted in Fig. 3. The first step, shown on the left hand side of the figure, involves the preparation of the qubits in an initial state for the computation.

The second step consists of a sequence of instructions, each associated with the implementation of a gate from a universal gate set to approximate a desired $N$-qubit unitary operation. Just as classical computing modifies the values of binary bits through logic gates (AND, OR, XOR, etc.) quantum computing evolves the state of entangled qubits through analogous quantum gates to achieve an algorithmically determined outcome (Figs. 466). Most systems are based on 1,2, or 3 qubit gates with $T$ (a $\pi / 8$ rotation gate, or $R_{3}$ ), Hadamard $(H)$ and CNOT forming the Universal set (see Fig. 5). These gates perform unitary operations on the qubits, corresponding to modifications of the phases of the complex numbers associated with the superposition state. For a single qubit, these unitary transformations can be represented as rotations on a sphere depicting the complex coefficients $a_{0}$ and $a_{1}$ in the state equation $|\Psi\rangle=a_{0}|0\rangle+a_{1}|1\rangle$ as shown in Fig.7 Any quantum algorithm then involves such a series of such unitary transformations. Though in Fig. 3 we depict quantum gates acting on single qubits and neighboring qubits only, the connectivity between qubits, or equivalently our 
ability to perform operations on distant qubits can substantially increase the efficiency of a quantum algorithm. The complexity of the quantum algorithm is given by the number of simple operations needed for each of the three steps. Typically, this complexity is dominated by the number of elementary unitary gates needed to prepare the initial state and the second step, since the complexity of simple measurements is assumed to be, at most, linear in the number of operations.

The final step is a measurement or read-out of the final state of the computation. As noted above, the nature of superposition means that this read-out is inherently probabilistic. Any measurement returns only $N$ bits of information from a $\mathrm{N}$-qubit quantum system. Algorithms are therefore designed to achieve high probability of producing the desired result in a way that does not depend on the problem size $N$. The way that quantum algorithms are written, they either provide a high probability of producing the correct answer, which is verifiable at low computational cost (e.g., checking prime factors of a number), or inherently are designed to compute a probability distribution (e.g., Monte Carlo sampling).

The literature is rich with a wide variety of algorithms that exhibit quantum speedup. Examples include matrix manipulations, the solution of sparse linear equations, quantum Monte-Carlo problems, classical simulated annealing problems and much more. For a catalog of a large number of popular algorithms see the "quantum zoo" maintained at NIST by Jordan [59]. There are many classical problems that are already utilizing such algorithms. Some others can exhibit speedup only for very large number of qubits. As a broad estimate, in order to perform a computation on a quantum computer that cannot be performed on a classical computer, we need to have at least 50 qubits. To implement a particular algorithm might require many more qubits than this, however.

In the cleanest form, a quantum computer "outperforming" its classical counterpart should be characterized by superpolynomial speedup over the best possible classical algorithm [78]. A term coined by Preskill [79] and used often in this context is the notion of Quantum Supremacy. This refers to a situation where a quantum computer markedly and demonstrably outperforms the fastest classical computer, at least for one specific demonstration problem (in general, irrespective of the usefulness of that problem). With this power, the opportunity is to identify potential problems that are intractable on today's leading classical computers, but could potentially be solved on a quantum computer in the order of minutes! Here again we should emphasize that the gain is not in how fast an individual operation is performed it is in how the number of operations required to implement a particular algorithm scale with the size of the problem being solved. This is harder to demonstrate when the problems are small-scale, but has the potential to be completely transformative for large-scale high-performance computing problems. We note that there is a growing preference in the literature to move towards a focus on useful applications where a quantum computer can outperform classical computation, providing a practical quantum advantage. 


\section{Implementation of Digital Quantum Computing}

Some of the properties that make quantum computing powerful also lead to difficulties for practical computing. In order to understand the requirements to achieve a practical quantum computing capability, we first need to understand the unique challenges faced in building a quantum computer. This requires: (1) isolating qubits from their environment, so that they can remain in superposition states during the calculation, avoiding so-called "decoherence" in which they are inadvertently perturbed by the environment; and (2) developing the high degree of control required in order to implement quantum gate operations. Development and implementation of quantum computing technology is ongoing, combining hardware for quantum computing, with simultaneous work on developing quantum algorithms (and indeed a full software stack for programming and verifying quantum computers) [80, 81]. In this section we survey the general challenges and state-of-the-art.

\section{A. Decoherence and Error Correction}

As with storage and processing of information in classical computers, operations on physical qubits are not necessarily perfect. Treatment of noise in quantum computers is not as easy as that in classical computers. The superposition can be altered due to noise, or unwanted coupling of the qubits to the surroundings. Moreover, most qubits are formed from two states of a system that can have more than two states (physical systems have more than 2 levels), so there can be problems with leakage to additional levels. These processes can lead to a disturbance of the so-called "coherent" superposition of different states stored in a quantum computer, which is referred to as "decoherence." This combined with imperfect implementation of operations that change the state of qubits cause errors that accumulate in time. A major building block in the development of gate-based quantum computers was the realization that these errors can be corrected. These quantum error correction (QEC) techniques are very similar to error correction codes for storage, processing and communication in classical computers and communications systems [79]. With QEC, one can effectively store information in multiple coordinated physical qubits, in what is often referred to as a logical qubit. These have some redundancy in the information they carry such that when they are affected, the errors can be corrected. When operating with a system that should be entirely error corrected, one must distinguish between the number of physical and logical (error corrected) qubits. Most manufacturers normally list the former, with no means of error correction.

Another question is how much we can compute if errors are not completely corrected. At the moment, when we have relatively few qubits available we can ask how the computational power of the system depends on the number of qubits and the error rate. The lower the error rate, the more complex an algorithm we can implement reliably on the hardware. This is often characterized using the notion of "gate depth," essentially the minimum number of operations in sequence that are required to implement a particular calculation on a given set of hardware. The larger the gate depth that can be reliably implemented, and the more qubits the system has, the more computational power we are able to draw from the system. 
In determining the capabilities of a quantum computer, we need to consider not just the number of qubits, but also the rate of errors (including decoherence) for qubits and gate operations. In cases where it is not possible to perform operations involving an arbitrary choice of qubits - but rather, gates can only be applied to certain combinations of qubits - then we also have to consider the connectivity of the system.

One way of quantifying the rate of errors is by considering the quantum volume, as shown in Fig. 8[82]. This figure represents the useful computing that can be done with a quantum computer (or the size of the space of problems that can be addressed), taking into account the number of available qubits, their connectivity, and the error rate [83]. Beyond a certain level (which depends on the error rate), it does not help in practical terms to add more qubits unless we can decrease the error rates at the same time. These linked challenges - scaling up the number of qubits while decreasing error rates - are being addressed across a range of hardware architectures for practical quantum computers.

\section{B. Hardware for Universal Quantum Computing}

The QC community as a whole has an overarching goal to build a general purpose, universal quantum computer that is error corrected, tolerates noise and remains coherent for the duration of computation: the digital universal quantum computer [84]. Google, IBM, Intel, Microsoft, and a large and growing number of start-ups in the US and abroad are focused on the development of such a machine. Quantum computers are increasingly available in the cloud, with commercial offerings from IBM, Microsoft, and Amazon recently being announced. Although these machines are not yet error corrected, metrics such as IBM's quantum volume show steady progress in increasing system size while simultaneously reducing sources of errors [85]. In parallel, Google claims that their latest 53 qubit machine has achieved quantum supremacy, in which the machine's coherence and size are sufficient that for a specially designed calculation, confirming the results of its calculations on a supercomputer is impractical [86]. As noted above, the most prevalent current technology for construction of quantum computing hardware consists of superconducting circuits, trapped ions, silicon quantum electronics, neutral atoms, quantum dots, topological systems based on Majorana Fermions, photonic networks, electron spins, and nuclear spins in the liquid or solid state. At the moment, it is not clear which of these platforms will ultimately provide the best technology platform for quantum computing. Following from the discussion of noise and decoherence in the previous section, the total number of qubits is not the only issue to be considered in evaluating progress in this direction. The connectivity, coherence, error rates, and therefore the capability to run algorithms for long durations are equally important. The same goes for the number of logical quantum gates and the depth and width of the quantum circuits required to execute an algorithm. Presently, the most advanced platforms in terms of increasing numbers of qubits and reducing error rates are superconducting circuits and trapped ions. We will briefly survey the progress in these directions as an example of the state-of-the-art.

Industry in the United States has invested particularly heavily in superconducting circuit technologies. The advantage of these qubits is that the technology can be integrated with existing electronics and, in principle, scaled up without 
too much inconvenience, and the individual qubits are fast. Progress is measured by the error rates of a qubit, the inter-qubit activity, the total number of the qubits, and the errors caused by the gates. IBM has released a 20-qubit superconducting curcuit based quantum computer ${ }^{5}$ They have also made several IBM Q quantum devices available for public use through the cloud including 5, 16, and 20 qubit devices accessible through the IBM-Qiskit, and IBM Q Network. IBM has moved towards 49 qubits 6 [87, 88]. Google is aiming to build a universal quantum computer of 72 qubits 78 [89, 90]. The start-up company Rigetti has built a 19-qubit computer. They have tested it on a machine learning task 9 [91]. Now they have two computers with 8 and 19 qubits available for cloud computing 10 , and are working on a 128-qubit chip ${ }^{11}$ [92]. Intel has released a 49-qubit quantum computer ${ }^{12}[13$, and is continuing work towards the development of newer chips ${ }^{14}$. The challenges for superconducting qubits lie in making the qubits identical, reducing errors in individual gate operations, and improving the connectivity. Because these qubits are usually coupled capacitively, it is a difficult engineering issue to make all-to-all couplings in these systems.

The other platform that is particularly advanced involves the use of trapped arrays of individual atomic ions. There, the qubits are written by manipulating the electronic states of ions with lasers, and quantum gates are performed using Coulomb interactions between ions in the array. The leading developers of this platform include startup companies in Maryland (IonQ) and in Austria (Alpine Quantum Technologies). In the United Kingdom, there is a national quantum technology hub that is focused around the development of quantum computers with trapped ions. These platforms have demonstrated in excess of 20 qubits with two-qubit gates that are currently higher fidelity than superconducting circuits. These systems allow for the possibilities of scaling using optical interfaces to link small quantum computers. While the operation times are slower, the coherence times of these systems are very long, allowing for a particularly high gate depth. In addition, the connectivity is generally excellent: it is more straight-forward to perform operations between selected qubits in a large array in this implementation, which doesn't require hardware for coupling specific qubits, as superconducting qubits do. While it is straight-forward to add additional identical qubits to the system (as the ions are naturally identical), there are physical limitations to the number of ions that interact with each other in a regular trap (because the interactions rely on normal modes of the collective motion of the ions, which become closer in frequency

\footnotetext{
${ }^{5}$ IBMq, Quantum Computing at IBM, 2018, https://www.research.ibm.com/ibm-q/learn/what-is-ibm-q/

${ }^{6}$ Edwin Pednault, Quantum Computing: Breaking Through the 49 Qubit Simulation Barrier, 2017, https://www.research.ibm.com/ibmq/learn/what-is-ibm-q/

${ }^{7}$ Google AI, Quantum AI, 2018, https://ai.google/research/teams/applied-science/quantum-ai

${ }^{8}$ Julian Kelly, A Preview of Bristlecone, Google's New Quantum Processor, 2018, https://ai.googleblog.com/2018/03/a-preview-of-bristleconegoogles-new.html

${ }^{9}$ Will Zeng, Unsupervised Machine Learning on Rigetti 19Q with Forest 1.2, 2017, https://medium.com/rigetti/unsupervised-machine-learningon-rigetti-19q-with-forest-1-2-39021339699

${ }^{10}$ Rigetti Computing,QPU Specifications, 2018, https://www.rigetti.com/qpu

${ }^{11}$ Chad Rigetti, The Rigetti 128-qubit chip and what it means for quantum, 2017, https://medium.com/rigetti/the-rigetti-128-qubit-chip-and-whatit-means-for-quantum-df757d1b71ea

${ }^{12}$ Intel, 2018 CES: Intel Advances Quantum and Neuromorphic Computing Research, 2018, https://newsroom.intel.com/news/intel-advancesquantum-neuromorphic-computing-research

${ }^{13}$ Intel, Quantum Computing, 2018, https://newsroom.intel.com/press-kits/quantum-computing/

${ }^{14}$ Intel, Intel Starts Testing Smallest 'Spin Qubit' Chip for Quantum Computing, 2018, https://newsroom.intel.com/news/intel-starts-testingsmallest-spin-qubit-chip-quantum-computing/
} 
when ions are added). This has led to the development of both segmented ion traps and architectures with the optical connections mentioned above, which allow for a moderate number of ions to be maintained in any one trap.

We note that there have been recent major advances in other candidate technologies, for example in using highlyexcited Rydberg states of neutral atoms to provide fast, high-fidelity gates [93]. These systems present fewer technical challenges for scaling to a few hundred qubits than other platforms, but until recently the gate fidelities have been limited by laser noise and coherence times are generally limited by the lifetime of the highly excited states. However, there has been an enormous increase in the number of groups taking this approach, due to significant improvements in gate fidelities, and the promising potential for scaling [36].

There have also been significant advances in silicon-based qubits and photonic quantum computing. Microsoft, together with several academic groups, is working on development of topological qubits, which provide another potential

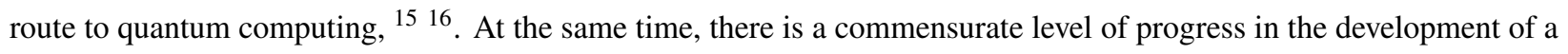
full software stack, ranging across architectures and algorithms for quantum computing. This includes the development of compilers for quantum programming (which would interface a generic language to specific hardware) ${ }^{17}$, building emulators for quantum computers on which algorithms can be tested. This software development often involves highly interdisciplinary research, with physicists, mathematicians, and computer scientists contributing substantially both in academia and in industry. Within industry, there are many quantum software start-up companies, as well as substantial groups at Microsoft, Intel, IBM, Google, Atos-Bull, and many other companies. There is a substantial growing interaction between researchers developing quantum algorithms and potential end-users, both in industry and in a diverse range of academic fields. This software stack also includes error correction mechanisms and verification techniques for quantum computers. None of the devices mentioned above have yet reached the so-called "threshold level" for error correction in which we can permanently protect quantum information, and errors currently restrict the gate depth of quantum algorithms that can be reliably implemented. Research is therefore ongoing towards improvement of QEC techniques to lower this threshold.

Overall, the progress in hardware and software for universal quantum computation is very substantial and promising for the future. At the same time, compared with the current state-of-the-art, the error rates need to be smaller and/or the system sizes larger in order to compete with classical high-performance computing even for the purposes of demonstration problems relevant to basic science. For many quantum algorithms, we will potentially require quantum computers with many thousands of error corrected qubits [94]. As a result, in parallel to the development of universal quantum computers, there is significant interest in taking advantage of short to medium term opportunities presented by

\footnotetext{
${ }^{15}$ Microsoft Quantum Team, The Microsoft approach to quantum computing, 2018, https://cloudblogs.microsoft.com/quantum/2018/06/06/themicrosoft-approach-to-quantum-computing/

${ }^{16}$ Microsoft Quantum Team, Developing a topological qubit, 2018, https://cloudblogs.microsoft.com/quantum/2018/09/06/developing-atopological-qubit/

${ }^{17}$ Microsoft Quantum Team, Atom by atom: Fabricating materials for a quantum computer, 2018, https://cloudblogs.microsoft.com/quantum/2018/09/18/atom-by-atom-fabricating-materials-for-a-quantum-computer/
} 
these hardware platforms for solving specialized problems.

\section{Alternatives to Gate-Based Digital Quantum Computing}

It is safe to indicate that it is currently possible to conduct gate-based digital quantum computation with about 20 qubits with average gate error rates of order of a few percent. To achieve an advantage for quantum over classical computing, it is required to increase the number of qubits to about 50 (because for qubit numbers up to around $\sim 40$, it is possible to use large shared-memory computers to directly simulate quantum states of the corresponding quantum computers), and most estimates suggest we also need to decrease the error rates to less than $0.1 \%$, to enable a path towards fault-tolerant computing. This is expected to happen possibly within the next 5-10 years. In the meantime, other near-term alternatives might provide the fastest route to useful quantum computing.

One popular option is referred to as Noisy Intermediate-Scale Quantum (NISQ) Computing [9]. The devices for this computing paradigm are likely to consist of 100s of imperfect qubits, which can only operate for some fixed maximum number of clock cycles before their qubits decohere. The algorithms in these machines are based on digital gate operations, but without any QEC. There is currently substantial active research in the applicability of NISQ hardware to selected problems in optimization, machine learning, data compression, particle physics, chemistry, materials science and other scientific computations. An important element of this is the idea of using a quantum system as a co-processor to a calculation on a classical computer. Algorithms that are developed in this form are often referred to as hybrid quantum-classical computation (HQCC), and provide a promising route towards near-term quantum computing [60-62]. This type of integration is much in the spirit of current emerging classical exascale heterogeneous HPC hardware [95]. To a certain extent, the nature of quantum computing is that it is particularly good at certain difficult, specialized operations. So, it is likely (for many decades at least) that a combination of existing HPC with quantum computing will provide the optimal solution.

Another option involves the use of devices that operate as analogue computers, including quantum simulators, and quantum annealers. An analogue quantum computer, in general, is a device where operations are implemented as controlled interactions between qubits that operate continuously in time, rather than being broken into discrete quantum gates. These machines are not general purpose universal quantum computers, but can be designed to deal with specific problems and potentially provide significant quantum speedups. This is by control of the Hamiltonian to advance the quantum state of the system. The operation is conducted in an analog fashion; therefore, cannot be subject to QEC. A quantum simulator [63] usually has the specific goal of simulating a physical system and computing the properties of that

system. These are very interesting because they would make it possible to map other mathematical problems (especially in materials science, chemistry, and optimization) onto the problem of computing the evolution of a quantum mechanical system. There is significant interest in building quantum simulation architectures from neutral atoms (where the starting point is often an array of hundreds up to many thousands of atoms, but currently with much less programable control 
than we have over individual qubits in universal quantum computers), as well as trapped ions, photonic networks, and superconducting circuits. There is significant investment in this direction ( e.g., from the European Union's Quantum Technologies Flagship).

A quantum annealer is closely related, in that it is also an analogue device, but is generally built for a particular type of problem, in which the computation is conducted by using control Hamiltonians that change slowly in time. In this way, the ground state of the original Hamiltonian is transformed to the ground state of the final one, which is designed to encode the solution to some problem of interest. For example, optimization problems can generally be rewritten mathematically into problems to minimize the energy of a set of two-state systems, or "spins," with an energy penalty for spin configurations chosen in such a way as to encode the specific optimization problem [96]. With the correct connectivity, these problems of minimizing the energy of a spin system can be translated onto a quantum annealer.

Generally, hardware appropriate for quantum simulation with sufficient connectivity and control over the system parameters (programmability) are good candidate platforms for quantum annealing. The machines built by $\mathrm{D}$-Wave systems are examples of special-purpose quantum annealers with superconducting qubits. The D-Wave $2000 \mathrm{Q}$ is a commercially available quantum annealer containing 2048 qubits 18 [65 specifically designed to solve the Ising spin model minimization problem, which is mathematically equivalent to the quadratic unconstrained binary optimization (QUBO) model minimization problem. In order to make use of this hardware for optimization, one must be able to recast the optimization problem at hand into an equivalent QUBO model problem.

Although significant progress has been made on demonstrating model optimization problems on the D-Wave system and other devices, the solution of large-scale problems that outperform classical algorithms is still to be demonstrated on quantum annealers. For some devices, the limitations involve combinations of the size, connectivity, and potentially the error rate of the hardware [70]. It is also broadly a non-trivial problem to define and detect quantum speedup for these analogue devices [66]. At the same time, exploring these approaches has led to substantial improvement in classical algorithms for optimization problems, as the energy of the spin encodings of the optimization problem can also be minimized using numerical techniques that were developed in physics specifically to investigate spin systems [97]. This provides further motivation to explore quantum computing solutions to new classes of problems, as it can also provide new ways to improve the existing classical algorithms.

\section{QC Prospects for Aeroscience and Engineering}

At the same time as major advances have been happening in quantum computing, classical supercomputing technology has also experienced significant growth, accompanied with development of massively parallel computers 19 The aeroscience and engineering communities have benefited from this growth, and are gearing up to take advantage

\footnotetext{
${ }^{18}$ DWave, Phase transitions in a programmable quantum spin-glass simulator, 2018, https://www.dwavesys.com/sites/default/files/qpt_synopsis.pdf

${ }^{19}$ For example, see Steve Lohr: Move Over, China: U.S. Is Again Home to World's Speediest Supercomputer, https://www.nytimes.com/2018/06/08/technology/supercomputer-china-us.html (2018)
} 
of exascale power in the near future. An example is in the field of computational fluid dynamics (CFD), where the projected rate of supercomputing growth is redefining the path towards future applications [71]. This head-start of classical technology seems to be a point of discussion within the community when forecasting the timetable for the relevance of quantum computing. With a modest speedup (low order polynomial), the crossover point at which quantum calculations beat equivalent classical calculations may be in the relatively far future. Classical hardware is likely to remain much faster and more highly parallel than quantum hardware for many years. It is possible that breakthroughs in quantum algorithms will lead to speedups sooner, but this is very difficult to predict. The overhead for quantum computing is also typically much higher than available in current classical computing technology [98]. Solving classical multi-dimensional non-linear partial differential equations, typical of those in CFD or other applications, on a digital universal quantum computer would generally require a fault-tolerant quantum computer, and could require millions of gates and qubits [94]. Again, typical estimates imply that it could be at least a decade, and most likely more before we have universal quantum computers at this scale.

The scenario portrayed above, does not imply that the community can only benefit from QC in the long run. As indicated already, quantum gates do not necessarily need to reach the fault tolerant threshold for us to conduct quantum computations. As an example, on NISQ Hardware, Variational quantum algorithms [99] belonging to the family of HQCC might be useful for solving differential equations. Moreover, analogue machine and quantum annealers have been effective for implementing quantum dynamics, which themselves are described by classes of differential equations. These machines are expected to be developed earlier than their digital quantum counterparts. If so, they could be useful for simulating some specific aspects of aeroscience and engineering if the differential equations of interest can be mapped into the form of the equations describing quantum dynamics. As noted above, we can also combine such NISQ computational capabilities with existing classical computers to produce a hybrid approach to computing that could have substantial advantages.

The key question for the aeroscience and engineering community is that of identifying which algorithms or applications will be impacted most effectively by advances in quantum computing. Clearly, as the early discovery of Shor's algorithm has demonstrated, areas such as cryptography stand to be revolutionized by quantum computing. However, developing algorithms that identify and use QC for any given purpose is a non-trivial challenge, and developing corresponding algorithms will require interactions between experts in algorithms and software for quantum computing, and specialists in computational aeroscience and engineering.

As a starting point, in recent years significant progress has been made in mapping some initial problems of interest in aerospace engineering onto quantum algorithms exhibiting theoretically predicted speedups. Below we summarize some of the initial advances in this direction, which will illustrate the substantial possibilities for the future. 


\section{A. Linear Equations and Machine Learning}

Linear algebra problems are ubiquitous in science and engineering, and they have been the subject of many quantum solution strategies. Whereas the solution of a sparse system of $N$ linear equations can be solved at best in $O(N)$ operations using classical algorithms, the recent HHL algorithm devised by Harrow et al. [47] runs in $O(\log N)$ time, providing an exponential quantum speedup. However, the output of this quantum algorithm is a quantum state with amplitudes proportional to the solution vector $x$ instead of the values of $x$ itself. This property makes the HHL algorithm useful for computing expectation values in $x$, i.e. $x^{T} M x$, where $M$ is some $N \times N$-dimensional matrix. Therefore, as with many other quantum algorithms, this approach is not directly applicable to all current linear algebra applications, at least in their current formulation.

Quantum algorithms for linear algebra problems are expected to be useful for both supervised and unsupervised machine learning; and general artificial intelligence applications. These applications rely on large data-sets often in high dimensional parameter space, with training outcomes that most often improve with increasing data-set size and dimensionality. Because of their ability to manipulate high-dimensional vectors using tensor-product spaces, quantum computing algorithms are expected to significantly impact the field of machine learning, which in turn has already found widespread applications throughout science and engineering. To this end, improved versions of the HHL algorithm will be paramount, especially when considering near-term implementations. One such improvement, given in Ref. [49], results in a quantum algorithm for systems of linear equations that requires a time that is logarithmic in one divided by the required precision, corresponding to an exponential speedup. Another improvement, given recently in Ref. [50], results in a quantum algorithm that needs only one ancillary qubit, in contrast to the many ancillary qubits needed by HHL. A small-scale demonstration of the latter is given in Ref. [100].

Machine learning techniques are also being used as indispensable tools in diverse areas such as decision making, autonomy, scheduling, model generation, chemical kinetics, and even turbulence modeling. The stochastic nature of machine learning and data-analytics problems are also well suited to benefit from quantum algorithmic approaches. For example, quadratic speedup of stochastic sampling problems has been demonstrated using quantum approaches [101], reducing the traditional Monte Carlo sampling convergence rate from $O(1 / \sqrt{M})$ to $O(1 / M)$, where $M$ is the number of samples. These approaches also have strong implications for uncertainty quantification techniques, which are increasingly becoming an integral part of many aerospace analysis and design problems.

\section{B. Differential Equations}

For aeroscience and engineering applications, numerical solution of differential equations is naturally very important, and some progress has been made in development of quantum algorithms for solving linear differential equations. Polynomial speedups over classical algorithms have been demonstrated in both finite-difference [51, 52] and finite element [53-55] discretization schemes. It is also demonstrated that differential equations can be represented in 
such a way that with $N$ variables, a quantum computer would need of order $\log N$ qubits, with an essentially linear time-complexity. The speedup becomes exponentially larger as the dimension of the problem increases with better performance obtained for the wave equation than for the heat equation [55]. Progress has also been made in the development of quantum algorithms for the solution of nonlinear differential equations [48, 51, 56, 57]. However, it is not yet clear whether an exponential speedup can be achieved; a polynomial speedup is much more likely.

Hybrid combinations of quantum and classical methods have also been effective for solving PDEs. An example is given in Ref. [53], where a classical preconditioning method is employed with a quantum algorithm. Other examples are the lattice gas model simulations of the diffusion [102] and Burgers' equation [103], and vortex in cell method solution of the Navier-Stokes equation [104, 105].

Another method which appears promising involves a "renormalization" algorithm [106]. This algorithm works by mapping the differential equation onto a tensor network [107, 108], and evolving the network towards the solution. These networks provide one of the most effective means of dealing with strongly correlated quantum systems [109, 110]. The matrix product states (MPS), an important subtype of tensor networks [111], appear very promising for numerical solution of differential equations. Another contribution with direct relevance to aerospace science $\&$ engineering is simulation of turbulent mixing [112] and chemically reactive flows [113] via an algorithm developed in quantum metrology [101]. These works exhibit a quadratic speedup over classical Monte Carlo methods for estimating moments of the probability density function (PDF). These results are very useful in Reynolds-averaged Navier Stokes simulation via PDF [114] and large eddy simulation (LES) via the filtered density function (FDF) [115, 116] methods.

\section{Current QC Communities}

It is true that currently, there are no quantum algorithms that can outperform their counterpart classical ones for commercial/practical applications. However, there are various user communities in which progress is being made in the use of both gate-based and analog quantum computers.

NASA's Quantum Artificial Intelligence Laboratory (QuAIL) at the NASA Ames Research Center was established to explore the potentials of quantum computing for NASA missions and computing requirements. NASA has also partnered with Google and USRA to host and make available subsequent generations of the D-Wave quantum annealer machine. The research program includes fundamental work in areas such as programming principles, hardware characterization, error correction and compilation techniques, as well as on-going efforts in specific application areas using quantum annealing. The QuAil team has investigated both theoretical and empirical aspects of quantum annealing for application areas including planning and scheduling, fault diagnosis and machine learning. Significant work has shown how problems from these application areas can be mapped onto a QUBO problem and solved on a quantum annealer. Hybrid algorithms with a combination of classical algorithms and quantum annealing tasks have also been demonstrated, and quantum heuristic algorithms are also being pursued and tested empirically. While in many cases these applications have 
demonstrated the potential to outperform classical approaches, significantly more capable hardware will be required to scale up to the problem sizes needed to achieve these goals. At the same time, although the program focuses on the most promising application areas for quantum computing, a significant gap exists between these applications and the majority of the current computational disciplines that consume the bulk of the resources on the NASA Pleiades classical supercomputer system.

D-Wave has also been collaborating with the USC-Lockheed QC center and the Quantum Institute at the Los Alamos National Laboratory promoting the use of their quantum annealers for various applications. As for gate-based QC, IBM, Microsoft, and Rigetti corporations have provided web access support with several quantum software developments for applications. With these communities, the hope is to demonstrate quantum supremacy in the near future. In the meantime, these efforts have been found to help in advancing classical computing algorithms as they result in viewpoints and intuition not previously conceived.

\section{Future Prospects}

Quantum computing clearly has the potential to be a disruptive technology that will revolutionize many current computational tasks affecting diverse areas of science and engineering. The rate of progress in QC technology has been very promising. In fact, the rapid developments in this technology has lead to what is now known as the "Neven's law," stating that: "quantum computers are gaining computational power relative to classical ones at a doubly exponential rate,"20 However, at the same time, for most problems, it is still unknown what advantages QC can deliver, at what cost, and within what time frame. The challenge for aerospace program planners is to formulate a suitable investment strategy that contributes to foundational advances in the field, enables researchers to stay abreast of national and international developments, and identifies opportunities for leveraging QC in specific application areas that are most relevant to aerospace missions. Important stakeholders need to maintain a pathfinder program that builds and maintains internal expertise in quantum computing, partners with relevant outside institutions, and is capable of making informed decisions on when opportunities are ripe for investment. Prescribing specific areas and levels of investment is necessarily a difficult and dynamic task at this early stage. However, the most logical approach is to focus on cross-cutting technological areas that are most relevant to the aerospace mission and current HPC application areas. In particular, there is a need to bridge the gap between current QC algorithms and the more traditional HPC application areas of relevance to aeroscience and engineering such as CFD, combustion, MDAO and materials.

The CFD2030 Vision [71] calls for the quantum computing demonstration of a relevant model problem (i.e. relevant to CFD) periodically over the next 15 years to assess the maturity of and guide investment decisions in QC for CFD applications. Of course, choosing a suitable model problem in itself can be a difficult task, given the disparities between quantum versus classical computing paradigms. However, fundamental new approaches to CFD, PDE-constrained

\footnotetext{
${ }^{20} \mathrm{xttps}$ ://www.quantamagazine.org/does-nevens-law-describe-quantum-computings-rise-20190618/
} 
optimization and uncertainty quantification (UQ) must be explored to answer these questions. At the same time, investigations of how known fundamental QC algorithms for linear algebra, optimization and sampling can be applied to existing HPC applications of national interest are also needed.

Engaging domain scientists and application researchers to participate in QC represents another fundamental challenge. Algorithmic research in QC is certainly a high risk endeavor, particularly for organizations or individuals with an application driven focus. Additionally, QC algorithms and paradigms are fundamentally different from the more familiar classical computing approaches. Therefore, simply making QC hardware and software available to established research groups may not be a viable strategy. Rather, a complete rethinking of the algorithmic approach will be required, including a thorough understanding of QC algorithmic paradigms, which in turn will require educational activities as well as interaction with other communities. Past computing paradigm shifts, such as the advent of vector computing in the 1970's and massively parallel computing in the 1990's were not nearly as disruptive, but were facilitated by new organizational structures.

At the national level, various federal government agencies manage significant and growing investments in quantum computing and more generally in quantum information science (QIS). As an example, the Networking and Information Technology Research and Development (NITRD) supplement to the President's 2017 budget contains no less than 50 references to QC/QIS and identifies on-going efforts at NIST, DOE, NSF and NASA, including highlights from the NASA QuAIL team work. Realizing the growing importance of QIS, an Interagency Working group (IWG) on Quantum Information Science has been formed under the Subcommittee on Physical Sciences of the National Science and Technology Council (NSTC) on Science (within the Office of Science and Technology Policy (OSTP)). The IWG contains membership from DoE, NSF, DoD NIST and IARPA and is tasked with assessing federal programs in QIS, monitoring the state of the field, providing a forum for inter-agency coordination and engaging in strategic planning of federal QIS activities and investments.

In order to ensure the maximum benefit, we also need a parallel community of future users asking what the key end applications might be, and also in the near term, what special purpose/NISQ machines can achieve. Many of the communities in the aeroscience and engineering are, to a large extent, disjoint from the condensed matter and quantum physics communities. However, we need to be aware of the ongoing progress; at least on a level where we can interface between fields and properly join the discussions of near and medium-term applications. To ensure this, we need to generate more interaction between aerospace scientists and engineers and QC researchers. We have reached a point in the development of QC and other quantum technologies where interaction and collaboration beyond a specific narrow scientific field is going to be a key to further developments. We need to develop strong engagement between people developing QC software and the potential end-users in aeroscience and engineering (CFD, for example). This implies connections to industry and to agencies such as NASA directly, and also engagement between physicists and engineers to identify what the most promising problems are that can be addressed in the short and medium term, and to ensure that 
the research in developing QC is directed towards these applications.

In order to identify the most promising areas and to enhance the likelihood of significant impact of QC on aeroscience and engineering in the relative near term, the community must:

- become aware of currently available QC algorithms that may be useful for specific applications. In doing so, critically examine the assumptions made in constructions of these algorithms. Some of the algorithms may demonstrate speedup but may be under assumptions that are not physical.

- engage directly with the community of researchers developing algorithms for quantum computers, through combinations of workshops and resulting research collaborations, making QC researchers aware of the most important computational problems relevant for aerospace.

- identify, in combination with QC researchers, problems that could be simulated with 100s-1000s of qubits and short depth quantum circuits; thus avoiding the need for quantum error correction techniques. Then implement these on QC devices that will be on the market in the near future.

- identify other areas where the governing equations can be mapped into a format that can be treated by quantum annealers and analogue simulators.

- Investigate other opportunities opened by quantum technologies: in addition to QC, there are important application areas in metrology, sensing and communication of relevance to aeroscience $\&$ engineering that will be impacted by advances in quantum technologies. Although these remain beyond the scope of this report, they should be considered in any future strategic planning exercises.

It is clear that the era of QC is here and the community is moving towards developments that could potentially have a profound impact on computational sciences and engineering. The challenge lies in understanding the new technology, and identifying the highest-impact applications. As the Nobel Laureate Bill Phillips said: “...Quantum information is a radical departure in information technology, more fundamentally different from current technology than the digital computer is from the abacus..." ${ }^{21}$ The CFD community along with other aeroscience and engineering communities need to recognize this evolution and understand that this is not a short-term endeavor. There is a need to build infrastructure and expertise in this technological frontier of the 21 st century. The response should not be crisis-driven. What we are seeing now in terms of global awareness of QC is the logical extension of what has been happening over the last 20 years.

\section{Acknowledgments:}

We are indebted to Drs. Andrew Childs, Michael Hatridge, Dieter Jaksch, Stephen Jordan, Jeremy Levy, Masoud Mohseni, Pedram Roshan, and Rolando Somma for very useful comments, discussions and input to this survey. The

\footnotetext{
${ }^{21}$ Carl J. Williams, Quantum Information Science: NIST's Role and the National Agenda, 2017, https://www.nist.gov/sites/default/files/documents/2017/05/09/Carl_VCAT_QIS_Final.pdf
} 
work of the first, third and fourth author was sponsored by NASA Transformational Tools and Technologies Project under the Transformative Aeronautics Concepts Program. The work of the second author is sponsored by EPSRC Grant No. EP/K000586/1. 


\section{References}

[1] Moore, G. E., “Cramming more components onto integrated circuits,” Electronics, Vol. 38, No. 8, 1965, pp. 1-4.

[2] Simonite, T., “Moore's Law Is Dead. Now What?” MIT Technology Review [online], https://www. technologyreview com/s/601441/moores-law-is-dead-now-what/. May 2016. [accessed Oct. 19 2018].

[3] Siegelmann, H. T., “Computation Beyond the Turing Limit," Science, Vol. 268, No. 5210, 1995, pp. 545-548. doi: 10.1126/science.268.5210.545.

[4] Lloyd, S., “Universal Quantum Simulators,” Science, Vol. 273, No. 5278, 1996, pp. 1073-1078. URL http://www . jstor. org/stable/2899535

[5] Simon, D. R., "On the Power of Quantum Computation," Society for Industrial and Applied Mathematics Journal on Computing, Vol. 26, No. 5, 1997, pp. 1474-1483.

[6] Milburn, G., "Quantum computation: Not the next step, but a whole new journey," Computing in Science and Engineering, Vol. 3, No. 6, 2001, pp. 87-93.

[7] Dowling, J. P., and Milburn, G. J., "Quantum technology: the second quantum revolution,” Philosophical Transactions of the Royal Society A: Mathematical, Physical and Engineering Sciences, Vol. 361, No. 1809, 2003, pp. $1655-1674$. doi:10.1098/rsta.2003.1227.

[8] Georgesc, I. M., Ashhab, S., and Nori, F., “Quantum Simulation,” Reviews of Modern Physics, Vol. 86, No. 1, 2014, pp. 153-185. doi:10.1103/RevModPhys.86.153.

[9] Preskill, J., "Quantum Computing in the NISQ era and beyond," Quantum, Vol. 2, 2018, p. 79. doi:10.22331/q-2018-08-06-79.

[10] Albash, T., and Lidar, D. A., "Adiabatic quantum computation,” Reviews of Modern Physics, Vol. 90, No. 1, 2018 , p. 015002. doi:10.1103/revmodphys.90.015002.

[11] Grumbling, E., and Horowitz, M. (eds.), Quantum Computing: Progress and Prospects, National Academies of Sciences, Engineering, and Medicine, Washington, D.C., 2019. doi:10.17226/25196.

[12] Martonosi, M., and Roetteler, M., "Next Steps in Quantum Computing: Computer Science’s Role," CoRR, Vol. abs/1903.10541, 2019. URL http://arxiv.org/abs/1903.10541

[13] Cho, A., "DOE pushes for useful quantum computing," Science, Vol. 359, No. 6372, 2018, pp. 141-142. doi:10.1126/science. 359.6372.141.

[14] Shor, P. W., “Algorithms for Quantum Computation: Discrete Logarithms and Factoring," Proceedings of the 35th Annual Symposium on Foundations of Computer Science, Institute of Electrical and Electronics Engineers Computer Society, Washington, DC, 1994, pp. 124-134. doi:10.1109/SFCS.1994.365700. 
[15] Shor, P. W., "Polynomial-Time Algorithms for Prime Factorization and Discrete Logarithms on a Quantum Computer," Society for Industrial and Applied Mathematics Review, Vol. 41, No. 2, 1999, pp. 303-332. doi:10.1137/S0036144598347011.

[16] Grover, L. K., “A Fast Quantum Mechanical Algorithm for Database Search,” Proceedings of the Twenty-Eighth Annual Association for Computing Machinery Symposium on Theory of Computing, Association for Computing Machinery, New York, NY, 1996, pp. 212-219. doi:10.1145/237814.237866.

[17] Kaye, P., Laflamme, R., and Mosca, M., An Introduction to Quantum Computing, Oxford University Press, USA, 2007.

[18] Nielsen, M. A., and Chuang, I. L., Quantum Computation and Quantum Information, Cambridge University Press, Cambridge, United Kingdom, 2010. 10 Years Anniversary Edition.

[19] Boixo, S., Isakov, S. V., Smelyanskiy, V. N., Babbush, R., Ding, N., Jiang, Z., Bremner, M. J., Martinis, J. M., and Neven, H., "Characterizing quantum supremacy in near-term devices," Nature Physics, Vol. 14, No. 6, 2018, pp. 595-600. doi:10.1038/s41567-018-0124-x.

[20] Neill, C., Roushan, P., Kechedzhi, K., Boixo, S., Isakov, S. V., Smelyanskiy, V., Megrant, A., Chiaro, B., Dunsworth, A., Arya, K., Barends, R., Burkett, B., Chen, Y., Chen, Z., Fowler, A., Foxen, B., Giustina, M., Graff, R., Jeffrey, E., Huang, T., Kelly, J., Klimov, P., Lucero, E., Mutus, J., Neeley, M., Quintana, C., Sank, D., Vainsencher, A., Wenner, J., White, T. C., Neven, H., and Martinis, J. M., “A blueprint for demonstrating quantum supremacy with superconducting qubits,” Science, Vol. 360, No. 6385, 2018, pp. 195-199. doi:10.1126/science.aao4309.

[21] Barends, R., Shabani, A., Lamata, L., Kelly, J., Mezzacapo, A., Heras, U. L., Babbush, R., Fowler, A. G., Campbell, B., Chen, Y., Chen, Z., Chiaro, B., Dunsworth, A., Jeffrey, E., Lucero, E., Megrant, A., Mutus, J. Y., Neeley, M., Neill, C., O’Malley, P. J. J., Quintana, C., Roushan, P., Sank, D., Vainsencher, A., Wenner, J., White, T. C., Solano, E., Neven, H., and Martinis, J. M., “Digitized adiabatic quantum computing with a superconducting circuit,” Nature, Vol. 534, No. 7606, 2016, pp. $222-226$. doi:10.1038/nature17658.

[22] Schoelkopf, R., "Quantum computing with superconducting circuits," 2016 Institute of Electrical and Electronics Engineers International Interconnect Technology Conference / Advanced Metallization Conference (IITC/AMC), Institute of Electrical and Electronics Engineers, 2016, pp. 43-44. doi:10.1109/iitc-amc.2016.7507674.

[23] Ofek, N., Petrenko, A., Heeres, R., Reinhold, P., Leghtas, Z., Vlastakis, B., Liu, Y., Frunzio, L., Girvin, S. M., Jiang, L., Mirrahimi, M., Devoret, M. H., and Schoelkopf, R. J., "Extending the lifetime of a quantum bit with error correction in superconducting circuits," Nature, Vol. 536, No. 7617, 2016, pp. 441-445. doi:10.1038/nature18949.

[24] Monroe, C. R., Schoelkopf, R. J., and Lukin, M. D., “Quantum Connections,” Scientific American, Vol. 314, No. 5, 2016, pp. 50-57. doi:10.1038/scientificamerican0516-50.

[25] Wang, C., Gao, Y. Y., Reinhold, P., Heeres, R. W., Ofek, N., Chou, K., Axline, C., Reagor, M., Blumoff, J., Sliwa, K. M., Frunzio, L., Girvin, S. M., Jiang, L., Mirrahimi, M., Devoret, M. H., and Schoelkopf, R. J., “A Schrodinger cat living in two boxes," Science, Vol. 352, No. 6289, 2016, pp. 1087-1091. doi:10.1126/science.aaf2941. 
[26] DiVincenzo, D. P., "Fault-tolerant Architectures for Superconducting Qubits," Physica Scripta, Vol. 2009, No. T137, 2009, p. 014020. doi:10.1088/0031-8949/2009/T137/014020.

[27] Geller, M., Pritchett, E., Sornborger, A., and Wilhelm, F., "Quantum Computing with Superconductors I: Architectures," Manipulating Quantum Coherence in Solid State Systems, NATO Science Series, Vol. 244, edited by M. Flatté and I. Tifrea, Springer Netherlands, 2007, pp. 171-194. doi:10.1007/978-1-4020-6137-0_5.

[28] Zhang, J., Pagano, G., Hess, P. W., Kyprianidis, A., Becker, P., Kaplan, H., Gorshkov, A. V., Gong, Z.-X., and Monroe, C., “Observation of a many-body dynamical phase transition with a 53-qubit quantum simulator," Nature, Vol. 551, No. 7682, 2017, pp. 601-604. doi:10.1038/nature24654.

[29] Linke, N. M., Maslov, D., Roetteler, M., Debnath, S., Figgatt, C., Landsman, K. A., Wright, K., and Monroe, C., "Experimental comparison of two quantum computing architectures," Proceedings of the National Academy of Sciences, Vol. 114, No. 13, 2017, pp. 3305-3310. doi:10.1073/pnas.1618020114.

[30] Debnath, S., Linke, N. M., Figgatt, C., Landsman, K. A., Wright, K., and Monroe, C., "Demonstration of a small programmable quantum computer with atomic qubits," Nature, Vol. 536, No. 7614, 2016, pp. 63-66. doi:10.1038/nature18648.

[31] Friis, N., Marty, O., Maier, C., Hempel, C., Holzäpfel, M., Jurcevic, P., Plenio, M. B., Huber, M., Roos, C., Blatt, R., and Lanyon, B., "Observation of Entangled States of a Fully Controlled 20-Qubit System," Physical Review X, Vol. 8, No. $2,2018$. doi:10.1103/physrevx.8.021012.

[32] Monz, T., Nigg, D., Martinez, E. A., Brandl, M. F., Schindler, P., Rines, R., Wang, S. X., Chuang, I. L., and Blatt, R., “Realization of a scalable Shor algorithm," Science, Vol. 351, No. 6277, 2016, pp. 1068-1070. doi:10.1126/science.aad9480.

[33] Leibfried, D., Blatt, R., Monroe, C., and Wineland, D., "Quantum Dynamics of Single Trapped Ions," Reviews of Modern Physics, Vol. 75, No. 1, 2003, pp. 281-324. doi:10.1103/RevModPhys.75.281.

[34] Blatt, R., and Wineland, D., "Entangled States of Trapped Atomic Ions,” Nature, Vol. 453, No. 7198, 2008 , pp. $1008-1015$. doi:10.1038/nature07125.

[35] Weiss, D. S., and Saffman, M., "Quantum computing with neutral atoms," Physics Today, Vol. 70, No. 7, 2017 , pp. 44-50. doi:10.1063/pt.3.3626.

[36] Saffman, M., "Quantum computing with atomic qubits and Rydberg interactions: progress and challenges," Journal of Physics B: Atomic, Molecular and Optical Physics, Vol. 49, No. 20, 2016, p. 202001. doi:10.1088/0953-4075/49/20/202001.

[37] Xia, T., Lichtman, M., Maller, K., Carr, A., Piotrowicz, M., Isenhower, L., and Saffman, M., "Randomized Benchmarking of Single-Qubit Gates in a 2D Array of Neutral-Atom Qubits,” Physical Review Letters, Vol. 114, No. 10, 2015. doi: 10.1103/physrevlett.114.100503.

[38] Barredo, D., de Léséleuc, S., Lienhard, V., Lahaye, T., and Browaeys, A., "An atom-by-atom assembler of defect-free arbitrary two-dimensional atomic arrays," Science, Vol. 354, No. 6315, 2016, pp. 1021-1023. doi:10.1126/science.aah3778. 
[39] Labuhn, H., Barredo, D., Ravets, S., de Léséleuc, S., Macrì, T., Lahaye, T., and Browaeys, A., “Tunable two-dimensional arrays of single Rydberg atoms for realizing quantum Ising models,” Nature, Vol. 534, No. 7609, 2016, pp. 667-670. doi:10.1038/nature18274.

[40] Hill, C. D., Peretz, E., Hile, S. J., House, M. G., Fuechsle, M., Rogge, S., Simmons, M. Y., and Hollenberg, L. C. L., “A surface code quantum computer in silicon," Science Advances, Vol. 1, No. 9, 2015, p. e1500707. doi:10.1126/sciadv.1500707.

[41] Hanson, R., Kouwenhoven, L. P., Petta, J. R., Tarucha, S., and Vandersypen, L. M. K., "Spins in Few-electron Quantum Dots," Reviews of Modern Physics, Vol. 79, No. 4, 2007, pp. 1217-1265. doi:10.1103/RevModPhys.79.1217.

[42] Awschalom, D., Loss, D., and Samarth, N. (eds.), Semiconductor Spintronics and Quantum Computation, Springer, Berlin, Germany, 2002.

[43] Weber, J. R., Koehl, W. F., Varley, J. B., Janotti, A., Buckley, B. B., de Walle, C. G. V., and Awschalom, D. D., “Quantum Computing with Defects," Proceedings of the National Academy of Sciences of the United States of America, Vol. 107, No. 19, 2010, pp. 8513-8518. doi:10.1073/pnas.1003052107.

[44] Prawer, S., and Greentree, A. D., "Diamond for Quantum Computing," Science, Vol. 320, No. 5883, 2008, pp. 1601-1602. doi:10.1126/science.1158340.

[45] Kane, B. E., “A Silicon-based Nuclear Spin Quantum Computer,” Nature, Vol. 393, No. 6681, 1998, pp. $133-137$. doi: $10.1038 / 30156$.

[46] Sparrow, C., Martín-López, E., Maraviglia, N., Neville, A., Harrold, C., Carolan, J., Joglekar, Y. N., Hashimoto, T., Matsuda, N., O’Brien, J. L., Tew, D. P., and Laing, A., "Simulating the vibrational quantum dynamics of molecules using photonics," Nature, Vol. 557, No. 7707, 2018, pp. 660-667. doi:10.1038/s41586-018-0152-9.

[47] Harrow, A. W., Hassidim, A., and Lloyd, S., "Quantum Algorithm for Linear Systems of Equations," Physical Review Letters, Vol. 103, No. 15, 2009, p. 150502. doi:10.1103/PhysRevLett.103.150502.

[48] Leyton, S. K., and Osborne, T. J., “A Quantum Algorithm to Solve Nonlinear Differential Equations,” arXiv preprint arXiv:0812.4423, Dec. 2008.

[49] Childs, A., Kothari, R., and Somma, R., "Quantum Algorithm for Systems of Linear Equations with Exponentially Improved Dependence on Precision,” SIAM Journal on Computing, Vol. 46, No. 6, 2017, pp. 1920-1950. doi:10.1137/16M1087072.

[50] Subasi, Y., Somma, R. D., and Orsucci, D., "Quantum Algorithms for Systems of Linear Equations Inspired by Adiabatic Quantum Computing," Physical Review Letters, Vol. 122, 2019, p. 060504. doi:10.1103/PhysRevLett.122.060504.

[51] Berry, D. W., "High-order quantum algorithm for solving linear differential equations," Journal of Physics A: Mathematical and Theoretical, Vol. 47, No. 10, 2014, p. 105301. doi:10.1088/1751-8113/47/10/105301. 
[52] Berry, D. W., Childs, A. M., Ostrander, A., and Wang, G., "Quantum algorithm for linear differential equations with exponentially improved dependence on precision,” Communications in Mathematical Physics, Vol. 356, No. 3, 2017, pp. 1057-1081. doi:10.1007/s00220-017-3002-y.

[53] Clader, B. D., Jacobs, B. C., and Sprouse, C. R., "Preconditioned quantum linear system algorithm," Physical Review Letters, Vol. 110, No. 25, 2013, p. 250504. doi:10.1103/PhysRevLett.110.250504.

[54] Montanaro, A., and Pallister, S., "Quantum algorithms and the finite element method," Physical Review A, Vol. 93, No. 3, 2016, p. 032324. doi:10.1103/PhysRevA.93.032324.

[55] Costa, P., Jordan, S., and Ostrander, A., “Quantum algorithm for simulating the wave equation,” arXiv preprint arXiv:1711.05394, Nov. 2017.

[56] Somma, R. D., Boixo, S., Barnum, H., and Knill, E., “Quantum Simulations of Classical Annealing Processes,” Physical Review Letters, Vol. 101, No. 13, 2008. doi:10.1103/physrevlett.101.130504.

[57] Papageorgiou, A., and Traub, J. F., "Quantum Algorithms for Continuous Problems and Their Applications," Advances in Chemical Physics, John Wiley \& Sons, Incorporated, 2014, pp. 151-178. doi:10.1002/9781118742631.ch06.

[58] Temme, K., Osborne, T. J., Vollbrecht, K. G., Poulin, D., and Verstraete, F., “Quantum Metropolis Sampling,” Nature, Vol. 471, No. 7336, 2011, pp. 87-90. doi:10.1038/nature09770.

[59] Jordan, S., “The Quantum Algorithm Zoo,” Catalog of quantum algorithms [online], https://math.nist.gov/quantum/ Zoo/, Apr. 2011. [accessed Oct. 19 2018].

[60] Li, J., Yang, X., Peng, X., and Sun, C.-P., "Hybrid Quantum-Classical Approach to Quantum Optimal Control," Physical Review Letters, Vol. 118, No. 15, 2017, p. 150503. doi:10.1103/PhysRevLett.118.150503.

[61] McClean, J. R., Romero, J., Babbush, R., and Aspuru-Guzik, A., “The Theory of Variational Hybrid Quantum-Classical Algorithms," New Journal of Physics, Vol. 18, No. 2, 2016, p. 023023. doi:10.1088/1367-2630/18/2/023023.

[62] Bauer, B., Wecker, D., Millis, A. J., Hastings, M. B., and Troyer, M., "Hybrid Quantum-Classical Approach to Correlated Materials," Physical Review X, Vol. 6, No. 3, 2016, p. 031045. doi:10.1103/PhysRevX.6.031045.

[63] Cirac, J. I., and Zoller, P., “Goals and Opportunities in Quantum Simulation,” Nature Physics, Vol. 8, No. 4, 2012, pp. $264-266$. doi:10.1038/nphys2275.

[64] Kokail, C., Maier, C., van Bijnen, R., Brydges, T., Joshi, M., Jurcevic, P., Muschik, C., Silvi, P., Blatt, R., Roos, C., and Zoller, P., “Self-Verifying Variational Quantum Simulation of Lattice Models,” Nature, Vol. 569, 2019, pp. 355-360. doi:10.1038/s41586-019-1177-4.

[65] Harris, R., Sato, Y., Berkley, A. J., Reis, M., Altomare, F., Amin, M. H., Boothby, K., Bunyk, P., Deng, C., Enderud, C., Huang, S., Hoskinson, E., Johnson, M. W., Ladizinsky, E., Ladizinsky, N., Lanting, T., Li, R., Medina, T., Molavi, R., Neufeld, 
R., Oh, T., Pavlov, I., Perminov, I., Poulin-Lamarre, G., Rich, C., Smirnov, A., Swenson, L., Tsai, N., Volkmann, M., Whittaker, J., and Yao, J., "Phase transitions in a programmable quantum spin glass simulator," Science, Vol. 361, No. 6398, 2018, pp. 162-165. doi:10.1126/science.aat2025, URL http://science.sciencemag.org/content/361/6398/162

[66] Ronnow, T. F., Wang, Z., Job, J., Boixo, S., Isakov, S. V., Wecker, D., Martinis, J. M., Lidar, D. A., and Troyer, M., "Defining and Detecting Quantum Speedup,”Science, Vol. 345, 2014, pp. 420-424. doi:10.1126/science.1252319.

[67] Figliola, P. M., "Federal Quantum Information Science: An Overview," Congressional Reseach Service [online], https: //fas.org/sgp/crs/misc/IF10872.pdf Jul. 2018. [accessed Oct. 19 2018].

[68] Aspuru-Guzik, A., van Dam, W., Farhi, E., Gaitan, F., Humble, T., Jordan, S., Landahl, A., Love, P., Lucas, R., Preskill, J., Muller, R., Svore, K., Wiebe, N., Williams, C., and Susut, C., “ASCR Report on Quantum Computing for Science,” Tech. rep., Quantum Computing Working Group, Department of Energy, 2015.

[69] Cramer, C., "Quantum Testbed Stakeholder Workshop," Slides [online], https://science.energy.gov/ /media/ascr/ ascac/pdf/meetings/201704/ASCAC_QTSW.pdf. Apr. 2017. [accessed Oct. 19 2018].

[70] Biswas, R., Jiang, Z., Kechezhi, K., Knysh, S., MandrÃ , S., O’Gorman, B., Perdomo-Ortiz, A., Petukhov, A., Realpe-GÃ³mez, J., Rieffel, E., Venturelli, D., Vasko, F., and Wang, Z., “A NASA Perspective on Quantum Computing: Opportunities and Challenges," Parallel Computing, Vol. 64, 2017,pp. 81-98. doi:10.1016/j.parco.2016.11.002.

[71] Slotnick, J., Khodadoust, A., Alonso, J., Darmofal, D., Gropp, W., Lurie, E., and Mavriplis, D., “CFD Vision 2030 Study: A Path to Revolutionary Computational Aerosciences," Tech. rep., NASA, March 2014. NASA/CR-2014-218178.

[72] Feynman, R. P., “Simulating Physics with Computers,” International Journal of Theoretical Physics, Vol. 21, No. 6-7, 1982, pp. $467-488$.

[73] Deutsch, D., "Quantum theory, the Church-Turing principle and the universal quantum computer," Proceedings of the Royal Society of London A, Vol. 400, No. 1818, 1985, pp. 97-117.

[74] Bernstein, E., and Vazirani, U., "Quantum Complexity Theory," Proceedings of the 25th Annual ACM Symposium on Theory of Computing, Association of Computing Machimery, 1993, pp. 11-20.

[75] Davis, M. (ed.), The Undecidable, Basic Papers on Undecidable Propositions, Unsolvable Problems and Computable Functions, Raven Press, New York, NY, 1965.

[76] Schumacher, B., “Quantum Coding,” Physical Review A, Vol. 51, No. 4, 1995, pp. 2738-2747. doi:10.1103/PhysRevA.51.2738.

[77] Dirac, P., "A new notation for quantum mechanics," Mathematical Proceedings of the Cambridge Philosophical Society, Vol. 35, No. 3, 1939, pp. 416-418.

[78] Papageorgiou, A., and Traub, J. F., "Measures of quantum computing speedup," Physical Review A, Vol. 88, No. 2, 2013, p. 022316. doi:10.1103/PhysRevA.88.022316. 
[79] Preskill, J., "Quantum Computing and the Entanglement Frontier,” arXiv preprint arXiv:1203.5813v3, Mar. 2012.

[80] Fingerhuth, M., Babej, T., and Wittek, P., “Open Source Software in Quantum Computing,” PLOS ONE, Vol. 13, No. 12, 2018, pp. 1-28. doi:10.1371/journal.pone.0208561, URL https://doi.org/10.1371/journal.pone.0208561

[81] Chong, F., Franklin, D., and Martonosi, M. R., "Programming Languages and Compiler Design for Realistic Quantum Hardware," Nature, Vol. 549, No. 7671, 2017, pp. 180-187. doi:10.1038/nature23459.

[82] Lirakis, C., "Quantum Computation," IBM-Q Group Lecture at Argonne Training Program on Extreme-Scale Computing (ATPESC) Workshop, DOE, Chicago, IL, 2017.

[83] Cross, A. W., Bishop, L. S., Sheldon, S., Nation, P. D., and Gambetta, J. M., "Validating quantum computers using randomized model circuits," Phys. Rev. A, Vol. 100, 2019, p. 032328. doi:10.1103/PhysRevA.100.032328, URL https: //link.aps.org/doi/10.1103/PhysRevA.100.032328

[84] Mohseni, M., Read, P., Neven, H., Boixo, S., Denchev, V., Babbush, R., Fowler, A., Smelyanskiy, V., and Martinis, J., “Commercialize Quantum Technologies in Five Years,” Nature, Vol. 543, No. 7644, 2017, pp. 171-174. doi:10.1038/543171a.

[85] Chow, J., and Gambetta, J., “Quantum Takes Flight: Moving from Laboratory Demonstrations to Building Systems,” , 2020. URL https://www .ibm.com/blogs/research/2020/01/quantum-volume-32/.

[86] Arute, F., Arya, K., Babbush, R., Bacon, D., Bardin, J. C., Barends, R., Biswas, R., Boixo, S., Brandao, F. G. S. L., Buell, D. A., Burkett, B., Chen, Y., Chen, Z., Chiaro, B., Collins, R., Courtney, W., Dunsworth, A., Farhi, E., Foxen, B., Fowler, A., Gidney, C., Giustina, M., Graff, R., Guerin, K., Habegger, S., Harrigan, M. P., Hartmann, M. J., Ho, A., Hoffmann, M., Huang, T., Humble, T. S., Isakov, S. V., Jeffrey, E., Jiang, Z., Kafri, D., Kechedzhi, K., Kelly, J., Klimov, P. V., Knysh, S., Korotkov, A., Kostritsa, F., Landhuis, D., Lindmark, M., Lucero, E., Lyakh, D., Mandrà, S., McClean, J. R., McEwen, M., Megrant, A., Mi, X., Michielsen, K., Mohseni, M., Mutus, J., Naaman, O., Neeley, M., Neill, C., Niu, M. Y., Ostby, E., Petukhov, A., Platt, J. C., Quintana, C., Rieffel, E. G., Roushan, P., Rubin, N. C., Sank, D., Satzinger, K. J., Smelyanskiy, V., Sung, K. J., Trevithick, M. D., Vainsencher, A., Villalonga, B., White, T., Yao, Z. J., Yeh, P., Zalcman, A., Neven, H., and Martinis, J. M., “Quantum supremacy using a programmable superconducting processor,” Nature, Vol. 574, No. 7779, 2019, pp. 505-510. doi:10.1038/s41586-019-1666-5, URL https://doi.org/10.1038/s41586-019-1666-5.

[87] Kim, M., “Google's quantum computing plans threatened by IBM curveball,” New Scientist, $2017 . \quad$ URL https://www .newscientist.com/article/2151032-googles-quantum-computing-plans-threatened-byibm-curveball/.

[88] Pednault, E., Gunnels, J. A., Nannicini, G., Horesh, L., Magerlein, T., Solomonik, E., and Wisnieff, R., "Breaking the 49-qubit barrier in the simulation of quantum circuits," arXiv preprint arXiv:1710.05867, Oct. 2017.

[89] Greene, T., "Google reclaims quantum computer crown with 72 qubit processor," The Next Web [online], https://tnw. to/ 2FmfU14. Mar. 2018. [accessed Oct. 19 2018]. 
[90] Conover, E., "Google moves toward quantum supremacy with 72-qubit computer," Science News, Vol. 193, No. 6, 2018, p. 13. URL https://wWW.sciencenews.org/article/google-moves-toward-quantum-supremacy-72-qubitcomputer

[91] Otterbach, J., Manenti, R., Alidoust, N., Bestwick, A., Block, M., Bloom, B., Caldwell, S., Didier, N., Fried, E. S., Hong, S., et al., "Unsupervised machine learning on a hybrid quantum computer," arXiv preprint arXiv:1712.05771, Dec. 2017.

[92] Knapp, A., and Konrad, A., "Rigetti Computing Takes Small Step Toward Cloud Services In Big Leap For Quantum Computing," Forbes [online], https://www. forbes.com/sites/alexknapp/2018/09/07/rigetti-computing-takessmall-step-toward-cloud-services-in-big-leap-for-quantum-computing/\#7ba6a7076503. Sep. 2018. [accessed Oct. 19 2018].

[93] Levine, H., Keesling, A., Omran, A., Bernien, H., Schwartz, S., Zibrov, A. S., Endres, M., Greiner, M., Vuletic, V., and Lukin, M. D., "High-Fidelity Control and Entanglement of Rydberg Atom Qubits," 2018. URL/http://arxiv.org/abs/1806. 04682

[94] Childs, A. M., Maslov, D., Nam, Y., Ross, N. J., and Su, Y., "Toward the first quantum simulation with quantum speedup,” Proceedings of the National Academy of Sciences, Vol. 115, No. 38, 2018, pp. 9456-9461. doi:10.1073/pnas.1801723115, URL http://wwW.pnas.org/content/115/38/9456

[95] Keyes, D., McInnes, L., Woodward, C., Gropp, W., Myra, E., Pernice, M., Bell, J., Brown, J., Clo, A., Connors, J., Constantinescu, E., Estep, D., Evans, K., Farhat, C., Hakim, A., Hammond, G., Hansen, G., Hill, J., Isaac, T., Jiao, X., Jordan, K., Kaushik, D., Kaxiras, E., Koniges, A., Lee, K., Lott, A., Lu, Q., Magerlein, J., Maxwell, R., McCourt, M., Mehl, M., Pawlowski, R., Randles, A., Reynolds, D., Rivière, B., Rüde, U., Scheibe, T., Shadid, J., Sheehan, B., Shephard, M., Siegel, A., Smith, B., Tang, X., Wilson, C., and Wohlmuth, B., "Multiphysics Simulations: Challenges and Opportunities," International Journal of High Performance Computing Applications, Vol. 27, No. 1, 2013, pp. 4-83. doi:10.1177/1094342012468181.

[96] Lucas, A., “Ising Formulations of Many NP Problems," Frontiers in Physics, Vol. 2, 2014, pp. 1-15. doi:10.3389/fphy.2014. 00005.

[97] Isakov, S. V., Zintchenko, I. N., Ronnow, T. F., and Troyer, M., “Optimised Simulated Annealing for Ising Spin Glasses," Computer Physics Communications, Vol. 192, 2015, pp. 265 - 271. doi:doi.org/10.1016/j.cpc.2015.02.015.

[98] Scherer, A., Valiron, B., Mau, S.-C., Alexander, S., van den Berg, E., and Chapuran, T. E., "Concrete Resource Analysis of the Quantum Linear-system Algorithm Used to Compute the Electromagnetic Scattering Cross Section of a 2d Target," Quantum Information Processing, Vol. 16, No. 3, 2017, pp. 1-65. doi:10.1007/s11128-016-1495-5.

[99] Peruzzo, A., McClean, J., Shadbolt, P., Yung, M.-H., Zhou, X.-Q., Love, P. J., Aspuru-Guzik, A., and O’Brien, J. L., “A variational eigenvalue solver on a quantum processor," Nature Communications, Vol. 5, 2014, p. 4213. doi:10.1038/ ncomms5213. 
[100] Wen, J., Kong, X., Wei, S., Wang, B., Xin, T., and Long, G., "Experimental Realization of Quantum Algorithms for a Linear System Inspired by Adiabatic Quantum Computing,” Physical Review A, Vol. 99, 2019, p. 012320. doi: 10.1103/PhysRevA.99.012320.

[101] Knill, E., Ortiz, G., and Somma, R. D., “Optimal Quantum Measurements of Expectation Values of Observables,” Physical Review A, Vol. 75, 2007, p. 012328. doi:10.1103/PhysRevA.75.012328.

[102] Berman, G. P., Ezhov, A. A., Kamenev, D. I., and Yepez, J., "Simulation of the Diffusion Equation on a Type-II Quantum Computer," Physical Review A, Vol. 66, No. 1, 2002, p. 012310. doi:10.1103/physreva.66.012310.

[103] Chen, Z., Yepez, J., and Cory, D. G., "Simulation of the Burgers Equation by NMR Quantum-information Processing," Physical Review A, Vol. 74, No. 4, 2006, p. 042321. doi:10.1103/physreva.74.042321.

[104] Steijl, R., and Barakos, G. N., "Parallel Evaluation of Quantum Algorithms for Computational Fluid Dynamics," Computers and Fluids, Vol. 173, 2018, pp. 22-28. doi:10.1016/j.compfluid.2018.03.080, URLhttp://eprints.gla.ac.uk/159789/

[105] Steijl, R., "Quantum Algorithms for Fluid Simulations," Quantum Dynamics and Computation, edited by V. N. Stavrou, IntechOpen, 2019. URL http://eprints.gla.ac.uk/187721/ a part of the simulation results presented were obtained using the EPSRCfunded ARCHIE-WeSt High Performance Computer (www.archie-west.ac.uk), EPSRC grant no. EP/K000586/1.

[106] Lubasch, M., Moinier, P., and Jaksch, D., "Multigrid Renormalization,” Journal of Computational Physics, Vol. 372, 2018 , pp. 587-602. doi:10.1016/j.jcp.2018.06.065.

[107] Orús, R., "A practical introduction to tensor networks: Matrix product states and projected entangled pair states," Annals of Physics, Vol. 349, 2014, pp. 117-158. doi:10.1016/j.aop.2014.06.013.

[108] Hastings, M. B., “An area law for one-dimensional quantum systems,” Journal of Statistical Mechanics: Theory and Experiment, Vol. 2007, No. 08, 2007, pp. P08024-P08024. doi:10.1088/1742-5468/2007/08/p08024.

[109] Lubasch, M., Joo, J., Moinier, P., Kiffner, M., and Jaksch, D., "Variational quantum algorithms for nonlinear problems," Physical Review A, Vol. 101, 2020, p. 010301. doi:10.1103/PhysRevA.101.010301.

[110] Al-Assam, S., Clark, S. R., and Jaksch, D., "The tensor network theory library," Journal of Statistical Mechanics: Theory and Experiment, Vol. 2017, No. 9, 2017, p. 093102. doi:10.1088/1742-5468/aa7df3.

[111] Schollwöck, U., "The density-matrix renormalization group in the age of matrix product states," Annals of Physics, Vol. 326, No. 1, 2011, pp. 96-192. doi:10.1016/j.aop.2010.09.012.

[112] Xu, G., Daley, A. J., Givi, P., and Somma, R. D., “Turbulent mixing simulation via a quantum algorithm,” AIAA Journal, Vol. 56, No. 2, 2018, pp. 687-699. doi:10.2514/1.J055896.

[113] Xu, G., Daley, A. J., Givi, P., and Somma, R. D., "Quantum Algorithm for the Computation of the Reactant Conversion Rate in Homogeneous Turbulence," Combustion Theory and Modelling, Vol. 53, No. 6, 2019, pp. 1090-1104. doi: $10.1080 / 13647830.2019 .1626025$. 
[114] Pope, S. B., Turbulent Flows, Cambridge University Press, Cambridge, United Kingdom, 2000.

[115] Givi, P., "Filtered Density Function for Subgrid Scale Modeling of Turbulent Combustion," AIAA Journal, Vol. 44, No. 1, 2006, pp. 16-23. doi:10.2514/1.15514.

[116] Livescu, D., Nouri, A. G., Battaglia, F., and Givi, P. (eds.), Modeling and Simulation of Turbulent Mixing and Reaction: For Power, Energy and Flight, Springer, Germany, 2020. doi:10.1007/978-981-15-2643-5. 


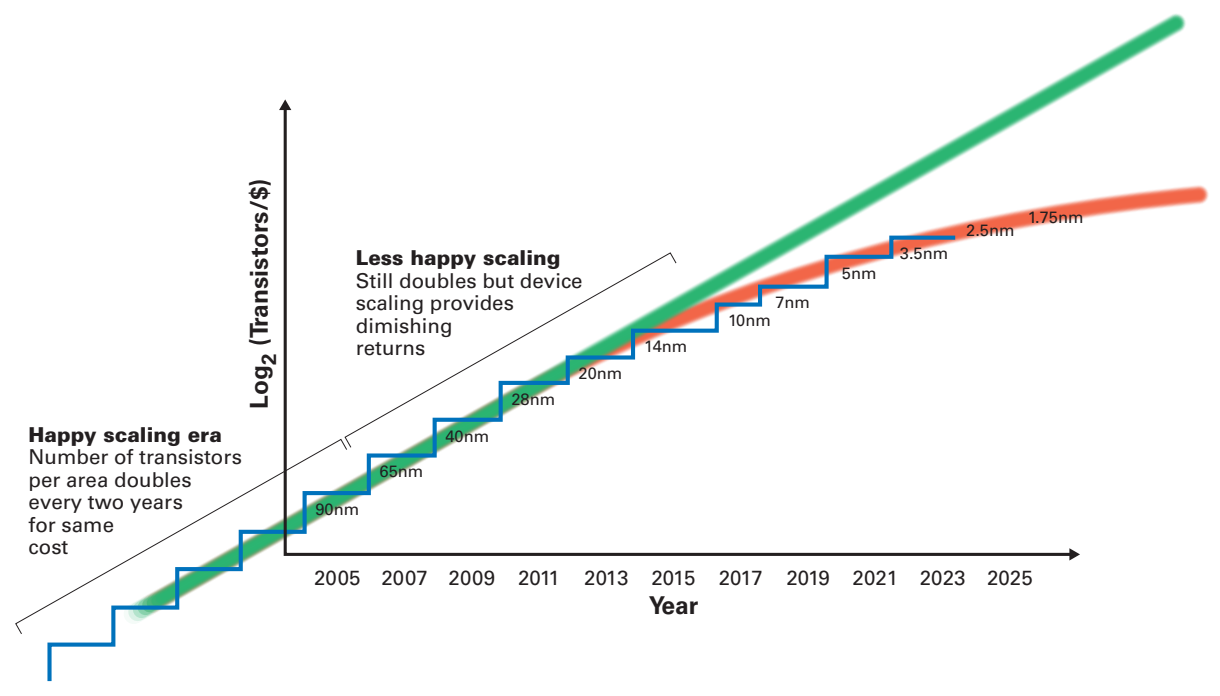

Fig. 1 Moore's law. From Ref. [2]. 


\section{Bit \\ (Classical Computing)}

0
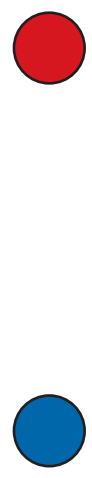

1
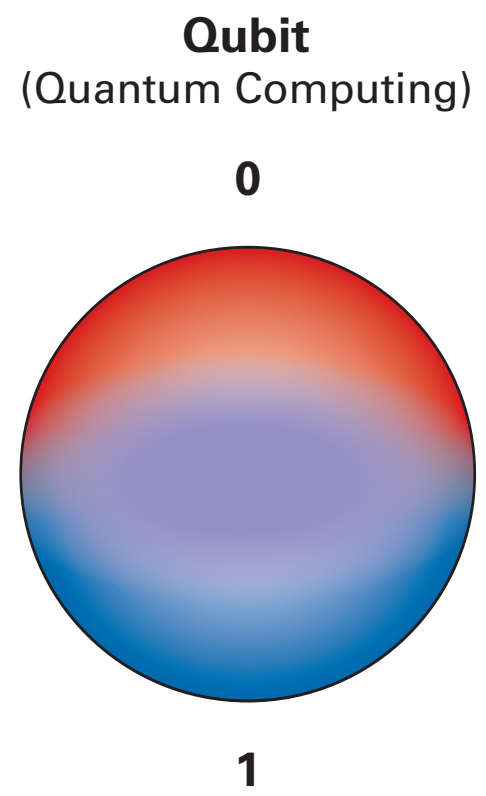

Fig. 2 Classical bit vs. quantum bit (qubit). 


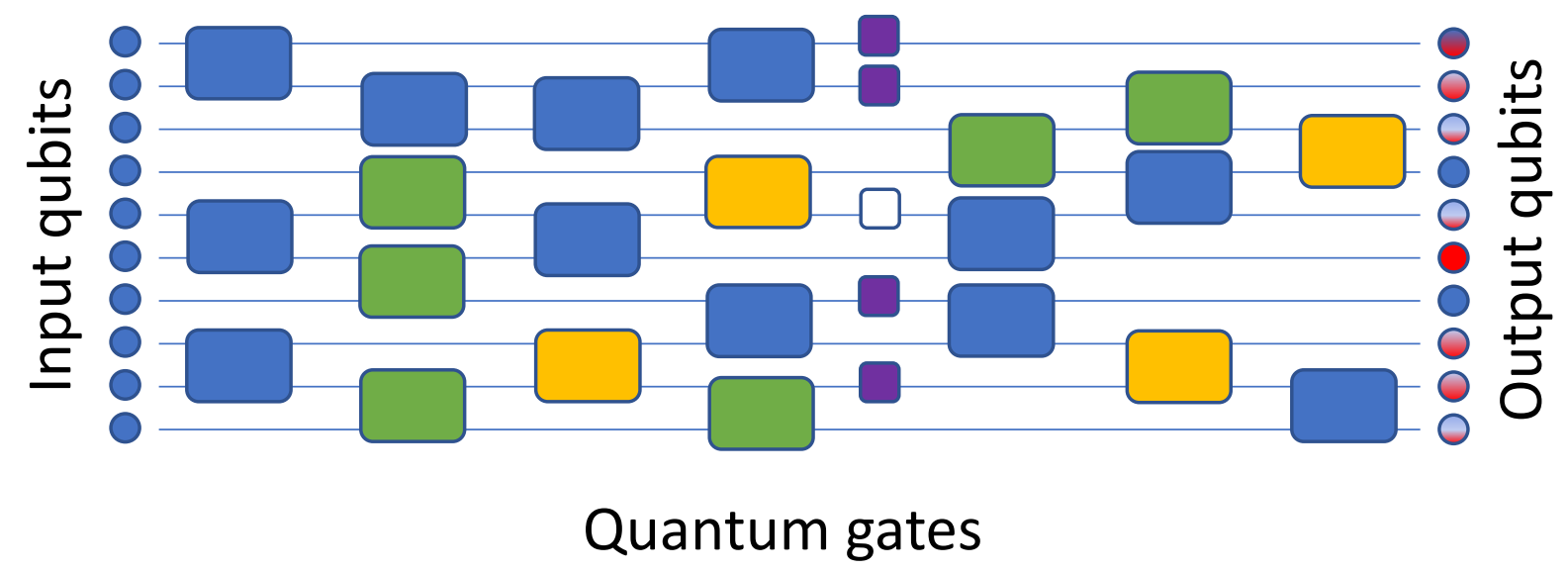

Fig. 3 Processing of information in a quantum computer 

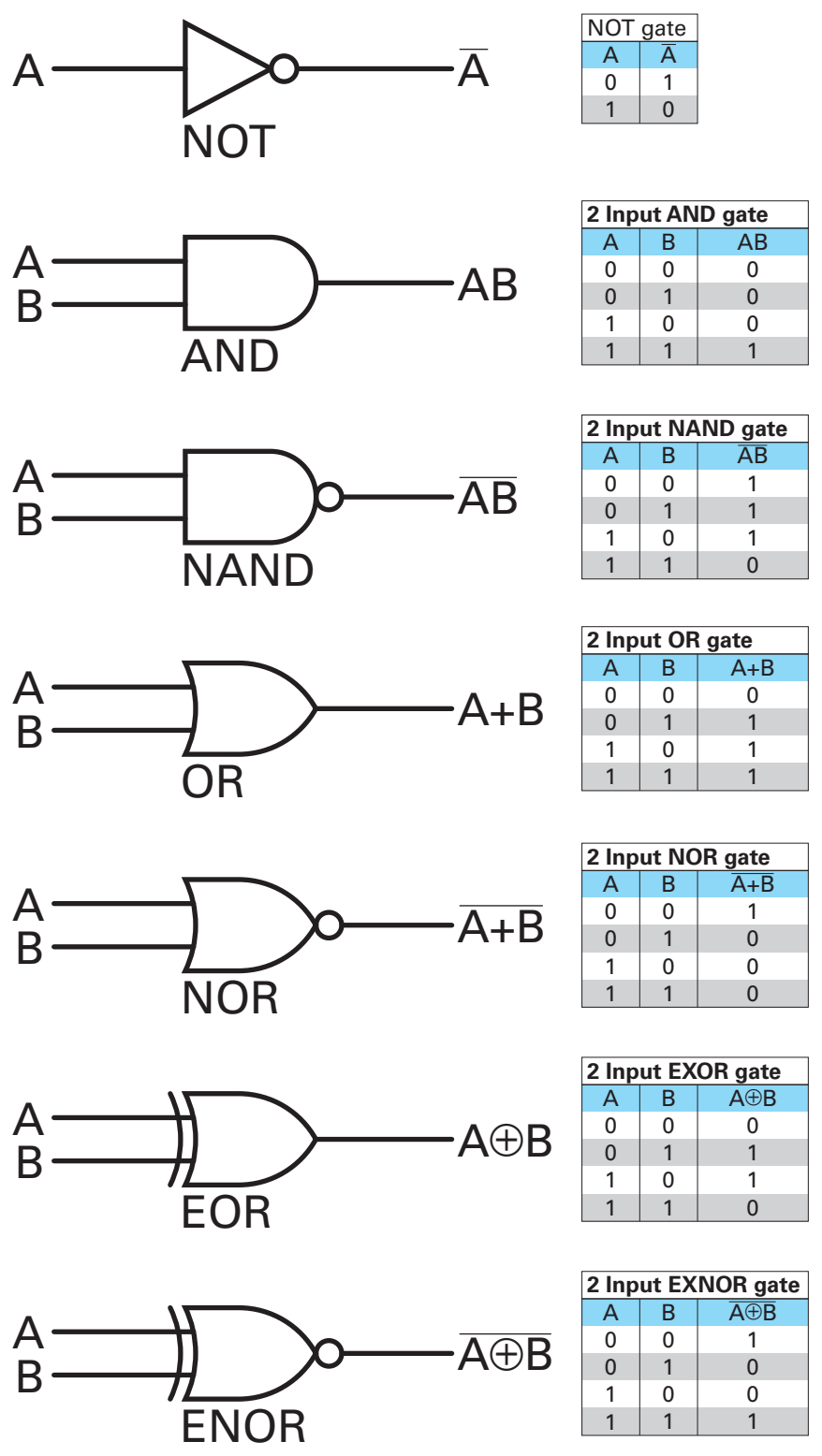

\begin{tabular}{|c|c|c|}
\hline \multicolumn{3}{|c|}{ Input EXNOR gate } \\
\hline $\mathrm{A}$ & $\mathrm{B}$ & $\overline{\mathrm{A}} \oplus \mathrm{B}$ \\
0 & 0 & 1 \\
0 & 1 & 0 \\
1 & 0 & 0 \\
1 & 1 & 1 \\
\hline
\end{tabular}

Fig. 4 Sample of classical logical gates. 


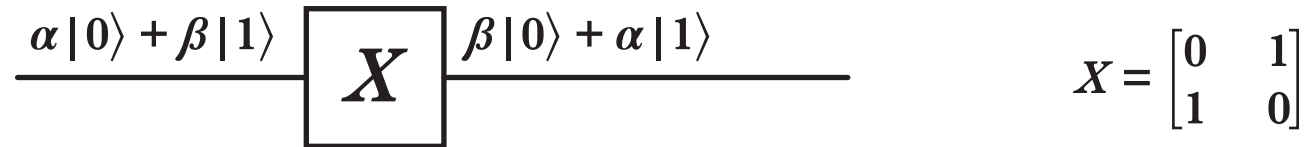

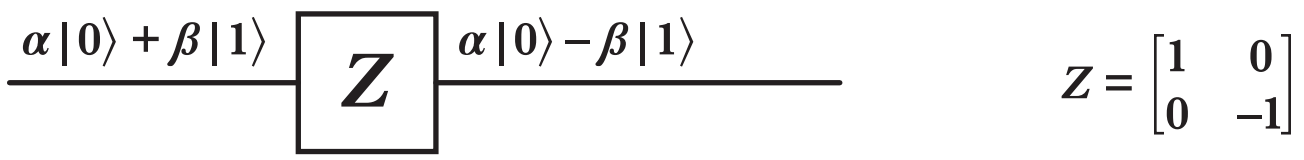

$$
\begin{aligned}
& \begin{array}{l}
\alpha|0\rangle+\beta|1\rangle \\
Y
\end{array} \quad Y=\left[\begin{array}{cc}
0 & -i \beta|0\rangle+i \alpha|1\rangle \\
i & 0
\end{array}\right] \\
& \underline{\alpha|0\rangle+\beta|1\rangle} H \frac{\alpha+\beta}{\sqrt{2}|0\rangle+\frac{\alpha-\beta}{\sqrt{2}}|1\rangle} \quad H=\frac{1}{\sqrt{2}}\left[\begin{array}{cc}
1 & 1 \\
1 & -1
\end{array}\right]
\end{aligned}
$$

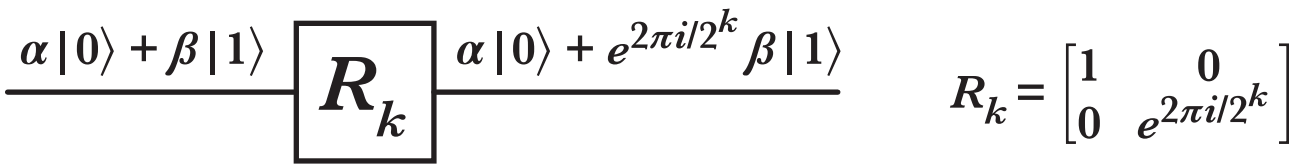

$$
\begin{aligned}
& |x\rangle-|x\rangle=U_{C N O T}=\left[\begin{array}{llll}
1 & 0 & 0 & 0 \\
0 & 1 & 0 & 0 \\
0 & 0 & 0 & 1 \\
0 & 0 & 1 & 0
\end{array}\right] \\
& |x\rangle \longrightarrow \quad|x\rangle \quad C R_{k}=\left[\begin{array}{cccc}
1 & 0 & 0 & 0 \\
0 & 1 & 0 & 0 \\
0 & 0 & 1 & 1 \\
0 & 0 & 0 & e^{2 \pi i / 2^{k}}
\end{array}\right]
\end{aligned}
$$

Fig. 5 Sample of quantum gates. 

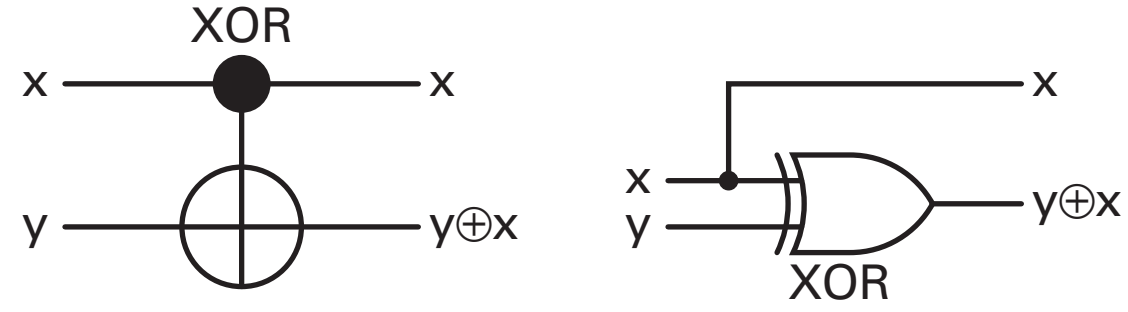

\begin{tabular}{|cc|cc|}
\hline \multicolumn{2}{|c|}{ Input } & \multicolumn{2}{c|}{ Output } \\
\hline$x$ & $y$ & $x$ & $y \oplus x$ \\
$|0\rangle$ & $|0\rangle$ & $|0\rangle$ & $|0\rangle$ \\
$|0\rangle$ & $|1\rangle$ & $|0\rangle$ & $|1\rangle$ \\
$|1\rangle$ & $|0\rangle$ & $|1\rangle$ & $|1\rangle$ \\
$|1\rangle$ & $|1\rangle$ & $|1\rangle$ & $|0\rangle$ \\
\hline
\end{tabular}

\begin{tabular}{|cc|cc|}
\hline \multicolumn{2}{|c|}{ Input } & \multicolumn{2}{c|}{ Output } \\
\hline $\mathrm{x}$ & $\mathrm{y}$ & $\mathrm{x}$ & $\mathrm{y} \oplus \mathrm{x}$ \\
0 & 0 & 0 & 0 \\
0 & 1 & 0 & 1 \\
1 & 0 & 1 & 1 \\
1 & 1 & 1 & 0 \\
\hline
\end{tabular}

Fig. 6 CNot gate. 


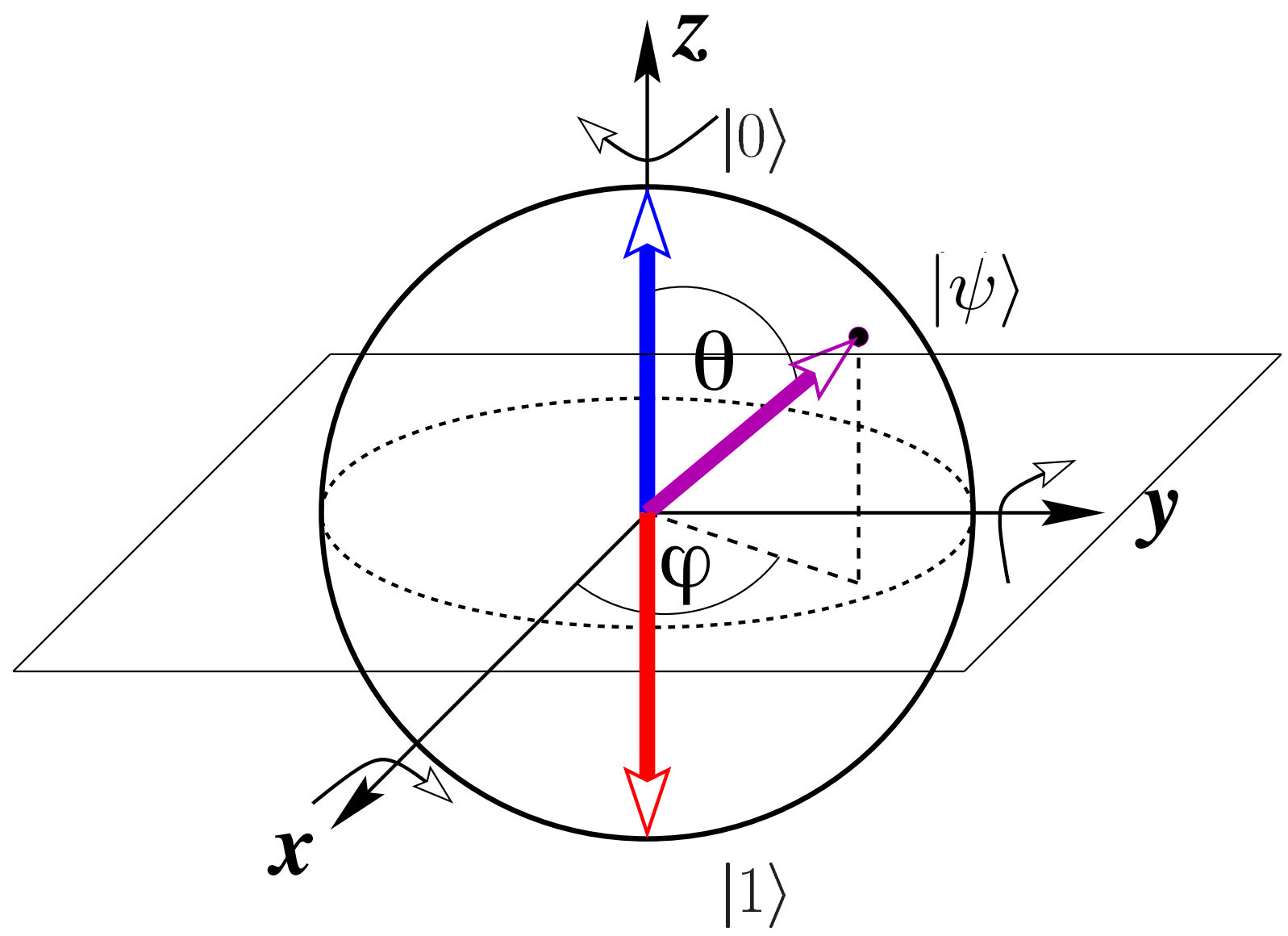

Fig. 7 A Bloch sphere representation of single-qubit unitary transformations. Up to a global phase factor, single qubit states can be represented as $|\Psi\rangle=\cos (\theta / 2)|0\rangle+e^{i \varphi} \sin (\theta / 2)|1\rangle$. The curved arrows indicate rotations with respect to the corresponding axis $v$. 


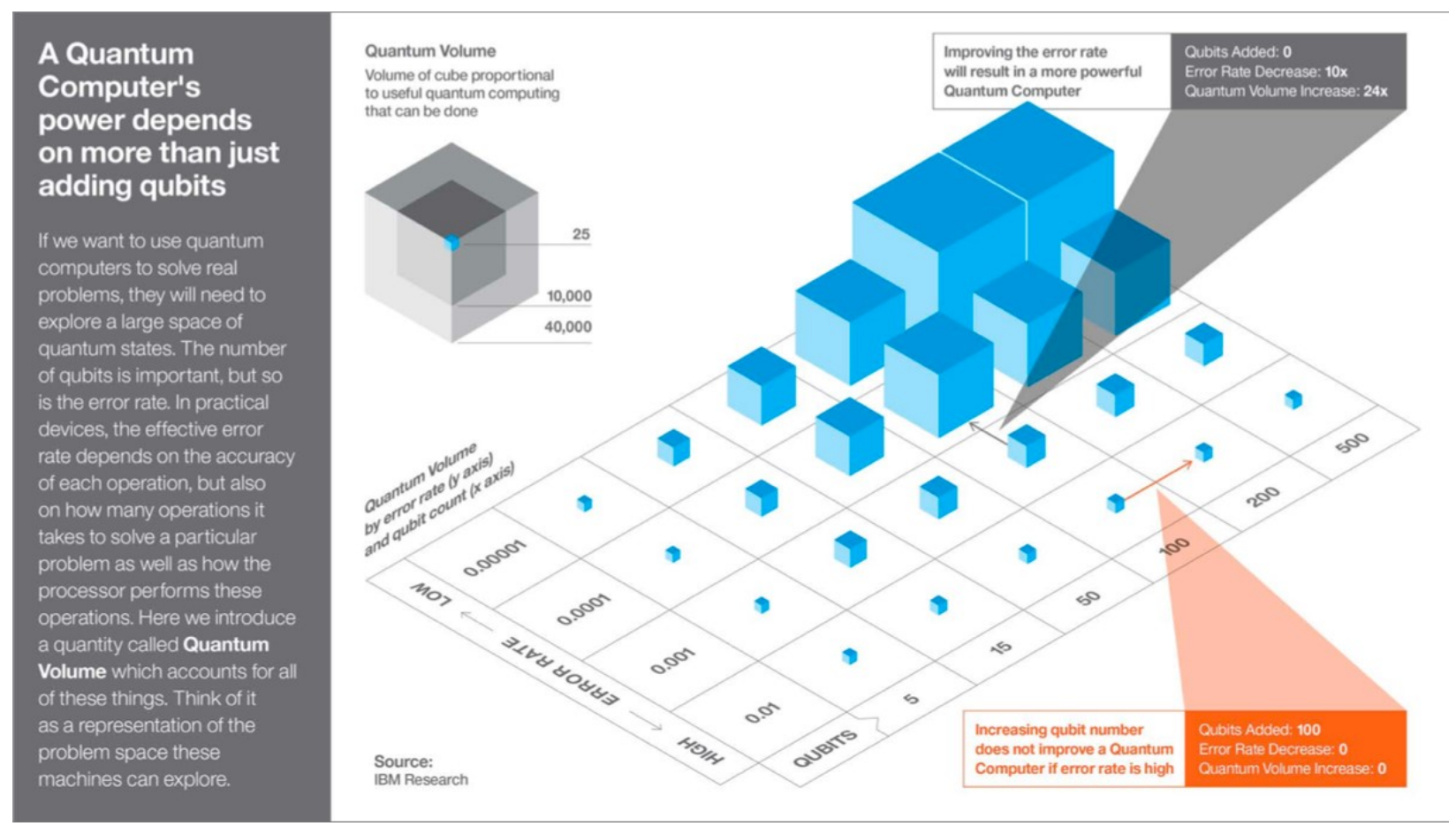

Fig. 8 Quantum Volume, which provides an estimate for the computational power of a quantum computer, accounting for the number of gates, connectivity, and error rates. From Ref. [82]. 